\title{
OPTIMAL ERROR ESTIMATES OF FINITE DIFFERENCE METHODS FOR THE GROSS-PITAEVSKII EQUATION WITH ANGULAR MOMENTUM ROTATION
}

\author{
WEIZHU BAO AND YONGYONG CAI
}

\begin{abstract}
We analyze finite difference methods for the Gross-Pitaevskii equation with an angular momentum rotation term in two and three dimensions and obtain the optimal convergence rate, for the conservative Crank-Nicolson finite difference (CNFD) method and semi-implicit finite difference (SIFD) method, at the order of $O\left(h^{2}+\tau^{2}\right)$ in the $l^{2}$-norm and discrete $H^{1}$-norm with time step $\tau$ and mesh size $h$. Besides the standard techniques of the energy method, the key technique in the analysis for the SIFD method is to use the mathematical induction, and resp., for the CNFD method is to obtain a priori bound of the numerical solution in the $l^{\infty}$-norm by using the inverse inequality and the $l^{2}$-norm error estimate. In addition, for the SIFD method, we also derive error bounds on the errors between the mass and energy in the discretized level and their corresponding continuous counterparts, respectively, which are at the same order of the convergence rate as that of the numerical solution itself. Finally, numerical results are reported to confirm our error estimates of the numerical methods.
\end{abstract}

\section{INTRODUCTION}

In this paper, we analyze different finite difference approximations of the GrossPitaevskii equation (GPE) with an angular momentum rotation term in $d$ dimensions $(d=2,3)$ for modeling a rotating Bose-Einstein condensate (BEC) 35, 12:

$$
\begin{aligned}
& i \partial_{t} \psi(\mathbf{x}, t)=\left[-\frac{1}{2} \nabla^{2}+V(\mathbf{x})-\Omega L_{z}+\beta|\psi(\mathbf{x}, t)|^{2}\right] \psi(\mathbf{x}, t), \\
& \mathbf{x} \in U \subset \mathbb{R}^{d}, \quad t>0,
\end{aligned}
$$

with the homogeneous Dirichlet boundary condition

$$
\psi(\mathbf{x}, t)=0, \quad \mathbf{x} \in \Gamma=\partial U, \quad t \geq 0,
$$

and initial condition

$$
\psi(\mathbf{x}, 0)=\psi_{0}(\mathbf{x}), \quad \mathbf{x} \in U .
$$

Here $t$ is time, $\mathbf{x}=(x, y)$ in two dimensions $(2 \mathrm{D})$, i.e., $d=2$, and resp., $\mathbf{x}=(x, y, z)$ in three dimensions (3D), i.e., $d=3$, are the cartesian coordinates, $U$ is a bounded computational domain, $\psi:=\psi(\mathbf{x}, t)$ is the complex-valued wave function, $\Omega$ is a dimensionless constant corresponding to the angular speed of the laser beam in

\footnotetext{
Received by the editor March 29, 2010 and, in revised form, March 26, 2011.

2010 Mathematics Subject Classification. Primary 35Q55, 65M06, 65M12, 65M22, 81-08.

Key words and phrases. Gross-Pitaevskii equation, angular momentum rotation, finite difference method, semi-implicit scheme, conservative Crank-Nicolson scheme.
} 
experiments, $\beta$ is a dimensionless constant characterizing the interaction (positive for repulsive interaction and negative for attractive interaction) between particles in the rotating BEC. $V(\mathbf{x})$ is a real-valued function corresponding to the external trap potential and it is chosen as a harmonic potential, i.e., a quadratic polynomial, in most experiments. $L_{z}$ is the $z$-component of the angular momentum defined as

$$
L_{z}=-i\left(x \partial_{y}-y \partial_{x}\right)=-i \partial_{\theta},
$$

where $(r, \theta)$ and $(r, \theta, z)$ are the polar coordinates in 2D and cylindrical coordinates in $3 \mathrm{D}$, respectively. In fact, since the first experimental realization of BEC in 1995 [5, 18, and the observation of quantized vortices in rotating BEC [1, 14, 32] which is related to superfluidity, theoretical studies of BEC and quantized vortices based on the above GPE have stimulated great research interests in quantum physics and applied and computational mathematics communities.

Extensive mathematical and numerical studies have been carried out for the above GPE (1.1) in the literature. Along the mathematical front, for the derivation, well-posedness and dynamical properties of the GPE (1.1) with (i.e., $\Omega \neq 0$ ) and without (i.e., $\Omega=0$ ) an angular momentum rotation term, we refer to [15, 23, 24, 29] and the references therein. In fact, it is easy to show that the GPE (1.1) conserves the total mass

$$
N(\psi(\cdot, t)):=\int_{U}|\psi(\mathbf{x}, t)|^{2} d \mathbf{x} \equiv N(\psi(\cdot, 0))=N\left(\psi_{0}\right), \quad t \geq 0,
$$

and the energy

$$
E(\psi(\cdot, t)):=\int_{U}\left[\frac{1}{2}|\nabla \psi|^{2}+V(\mathbf{x})|\psi|^{2}+\frac{1}{2} \beta|\psi|^{4}-\Omega \bar{\psi} L_{z} \psi\right] d \mathbf{x} \equiv E\left(\psi_{0}\right), \quad t \geq 0,
$$

where $\bar{f}$ denotes the conjugate of $f$. Along the numerical front, different efficient and accurate numerical methods including the time-splitting pseudospectral method [7, 25, 36, 37, finite difference method [2, 3], and Runge-Kutta or CrankNicolson pseudospectral method [14, 20] have been developed for the GPE without and with 6, 9, 11 the angular momentum rotation term. Of course, each method has its advantages and disadvantages. For numerical comparisons between different numerical methods for GPE without angular momentum rotation, or in a more general case, for the nonlinear Schrödinger (NLS) equation, we refer to [8, 17, 31, 39] and references therein.

Error estimates for different numerical methods of NLS, e.g. the GPE (1.1) without the angular momentum rotation $(\Omega=0)$ and/or $d=1$, have been established in the literatures. For the analysis of splitting error of the time-splitting or split-step method for NLS, we refer to [13, 19, 30, 33, 38, and the references therein. For the error estimates of the implicit Runge-Kutta finite element method for NLS, we refer to [4, 34. Error bounds of conservative Crank-Nicolson finite difference (CNFD) method for NLS in 1D was established in [16, 21, 22, 41]. In fact, their proofs for CNFD rely strongly on the conservative property of the method and the discrete version of the Sobolev inequality in 1D

$$
\|f\|_{L^{\infty}}^{2} \leq\|\nabla f\|_{L^{2}} \cdot\|f\|_{L^{2}}, \quad \forall f \in H_{0}^{1}(U) \text { with } U \subset \mathbb{R}
$$

which immediately imply an a priori uniform bound for $\|f\|_{L^{\infty}}$. However, the extension of the discrete version of the above Sobolev inequality is no longer valid in $2 \mathrm{D}$ and $3 \mathrm{D}$. Thus the techniques used in [16, 21] for obtaining error bounds 
of CNFD for NLS only work for conservative schemes in 1D and they cannot be extended to either high dimensions or nonconservative finite difference schemes. To our knowledge, no error estimates are available in the literature of finite difference methods for NLS either in high dimensions or for a non-conservative scheme. However, the GPE with the angular momentum rotation is either in 2D or 3D [6. 9, 11, 35. The main aim of this paper is to use different techniques to establish optimal error bounds of CNFD and the semi-implicit finite difference (SIFD) method for the GPE (1.1) with the angular momentum rotation in 2D and 3D. Based on our results, both CNFD and SIFD have the same second-order convergence rate in space and time. In our analysis, besides the standard techniques of the energy method, for SIFD, we adopt the mathematical induction; for CNFD, we first derive the $l^{2}$-norm error estimate and then obtain an a priori bound of the numerical solution in the $l^{\infty}$-norm by using the inverse inequality.

The paper is organized as follows. In section 2, we present SIFD and CNFD for the GPE with the angular momentum rotation and state our main error estimate results. In section 3, optimal error bounds of SIFD for GPE are established by using the energy method and the mathematical induction; and optimal error estimates of CNFD for GPE is built in section 4. In section 5, extensions of our results to other cases are discussed. In section 6 , numerical results are reported to confirm our error estimates. Finally, some conclusions are drawn in section 7 . Throughout the paper, we adopt the standard Sobolev spaces and their corresponding norms, let $C$ denote a generic constant which is independent of mesh size $h$ and time step $\tau$, and use the notation $p \lesssim q$ to represent that there exists a generic constant $C$ which is independent of time step $\tau$ and mesh size $h$ such that $|p| \leq C q$.

\section{Finite Difference Methods AND MAin RESUlts}

In this section, we introduce SIFD and CNFD methods for the GPE (1.1) in 2D on a rectangle $U=[a, b] \times[c, d]$, and resp., in $3 \mathrm{D}$ on a cube $U=[a, b] \times[c, d] \times[e, f]$, and state our main error estimate results.

2.1. Numerical methods. For the simplicity of notation, we only present the methods in 2D, i.e., $d=2$ and $U=[a, b] \times[c, d]$ in (1.1). Extensions to 3D are straightforward, and the error estimates in $l^{2}$-norm and discrete $H^{1}$-norm are the same in 2D and 3D. Choose time step $\tau:=\Delta t$ and denote time steps as $t_{n}:=n \tau$ for $n=0,1,2, \ldots$; choose mesh sizes $\Delta x:=\frac{b-a}{M}$ and $\Delta y:=\frac{d-c}{K}$ with $M$ and $K$ two positive integers and denote $h:=h_{\max }=\max \{\Delta x, \Delta y\}, h_{\min }:=\min \{\Delta x, \Delta y\}$ and grid points as

$$
x_{j}:=a+j \Delta x, \quad j=0,1, \ldots, M ; \quad y_{k}:=c+k \Delta y, \quad k=0,1, \ldots, K .
$$

Define the index sets

$$
\begin{aligned}
& \mathcal{T}_{M}=\{(j, k) \mid j=1,2, \ldots, M-1, k=1,2, \ldots, K-1\}, \\
& \mathcal{T}_{M}^{0}=\{(j, k) \mid j=0,1,2 \ldots, M, k=0,1,2 \ldots, K\} .
\end{aligned}
$$

Let $\psi_{j k}^{n}$ be the numerical approximation of $\psi\left(x_{j}, y_{k}, t_{n}\right)$ for $(j, k) \in \mathcal{T}_{M}^{0}$ and $n \geq 0$ and denote $\psi^{n} \in \mathbb{C}^{(M+1) \times(K+1)}$ be the numerical solution at time $t=t_{n}$. Introduce 
the following finite difference operators:

$$
\begin{aligned}
\delta_{x}^{+} \psi_{j k}^{n} & =\frac{1}{\Delta x}\left(\psi_{j+1 k}^{n}-\psi_{j k}^{n}\right), \quad \delta_{y}^{+} \psi_{j k}^{n}=\frac{1}{\Delta y}\left(\psi_{j k+1}^{n}-\psi_{j k}^{n}\right), \\
\delta_{t}^{+} \psi_{j k}^{n} & =\frac{1}{\tau}\left(\psi_{j k}^{n+1}-\psi_{j k}^{n}\right), \quad \delta_{x}^{-} \psi_{j k}^{n}=\frac{1}{\Delta x}\left(\psi_{j k}^{n}-\psi_{j-1 k}^{n}\right), \\
\delta_{y}^{-} \psi_{j k}^{n} & =\frac{1}{\Delta y}\left(\psi_{j k}^{n}-\psi_{j k-1}^{n}\right), \quad \delta_{t}^{-} \psi_{j k}^{n}=\frac{1}{\tau}\left(\psi_{j k}^{n}-\psi_{j k}^{n-1}\right), \\
\delta_{x} \psi_{j k}^{n} & =\frac{\psi_{j+1 k}^{n}-\psi_{j-1 k}^{n}}{2 \Delta x}, \quad \delta_{y} \psi_{j k}^{n}=\frac{\psi_{j k+1}^{n}-\psi_{j k-1}^{n}}{2 \Delta y}, \\
\delta_{t} \psi_{j k}^{n} & =\frac{\psi_{j k}^{n+1}-\psi_{j k}^{n-1}}{2 \tau}, \quad \delta_{x}^{2} \psi_{j k}^{n}=\frac{\psi_{j+1 k}^{n}-2 \psi_{j k}^{n}+\psi_{j-1 k}^{n}}{(\Delta x)^{2}} \\
\delta_{y}^{2} \psi_{j k}^{n} & =\frac{\psi_{j k+1}^{n}-2 \psi_{j k}^{n}+\psi_{j k-1}^{n}}{(\Delta y)^{2}}, \quad(j, k) \in \mathcal{T}_{M}, \\
\delta_{\nabla}^{+} \psi_{j k}^{n} & =\left(\delta_{x}^{+} \psi_{j k}^{n}, \delta_{y}^{+} \psi_{j k}^{n}\right), \quad \delta_{\nabla}^{2} \psi_{j k}^{n}=\delta_{x}^{2} \psi_{j k}^{n}+\delta_{y}^{2} \psi_{j k}^{n}, \\
L_{z}^{h} \psi_{j k}^{n} & =-i\left(x_{j} \delta_{y} \psi_{j k}^{n}-y_{k} \delta_{x} \psi_{j k}^{n}\right) .
\end{aligned}
$$

Then the conservative Crank-Nicolson finite difference (CNFD) discretization of the GPE (1.1) reads

$i \delta_{t}^{+} \psi_{j k}^{n}=\left[-\frac{1}{2} \delta_{\nabla}^{2}+V_{j k}-\Omega L_{z}^{h}+\frac{\beta}{2}\left(\left|\psi_{j k}^{n+1}\right|^{2}+\left|\psi_{j k}^{n}\right|^{2}\right)\right] \psi_{j k}^{n+1 / 2}, \quad(j, k) \in \mathcal{T}_{M}, n \geq 0$,

where

$V_{j k}=V\left(x_{j}, y_{k}\right), \quad \psi_{j k}^{n+1 / 2}=\frac{1}{2}\left(\psi_{j k}^{n+1}+\psi_{j k}^{n}\right), \quad(j, k) \in \mathcal{T}_{M}^{0}, \quad n=0,1,2, \ldots$

The boundary condition (1.2) is discretized as

$$
\psi_{0 k}^{n}=\psi_{M k}^{n}=0, \quad \psi_{j 0}^{n}=\psi_{j K}^{n}=0, \quad(j, k) \in \mathcal{T}_{M}^{0}, \quad n=0,1, \ldots,
$$

and the initial condition (1.3) is discretizaed as

$$
\psi_{j k}^{0}=\psi_{0}\left(x_{j}, y_{k}\right), \quad(j, k) \in \mathcal{T}_{M}^{0} .
$$

As proven in section 4, the above CNFD method conserves the mass and energy in the discretized level. However, it is a fully implicit method, i.e., at each time step, a fully nonlinear system must be solved, which may be very expensive, especially in $2 \mathrm{D}$ and $3 \mathrm{D}$. In fact, if the fully nonlinear system is not solved numerically to extremely high accuracy, e.g., at machine accuracy, then the mass and energy of the numerical solution obtained in practical computation are no longer conserved. This motivates us also consider the following discretization for the GPE.

The semi-implicit finite difference (SIFD) discretization for the GPE (1.1) is to use Crank-Nicolson/leap-frog schemes for discretizing linear/nonlinear terms, respectively, as

$$
i \delta_{t} \psi_{j k}^{n}=\left[-\frac{1}{2} \delta_{\nabla}^{2}+V_{j k}-\Omega L_{z}^{h}\right] \frac{\psi_{j k}^{n+1}+\psi_{j k}^{n-1}}{2}+\beta\left|\psi_{j k}^{n}\right|^{2} \psi_{j k}^{n}, \quad(j, k) \in \mathcal{T}_{M}, \quad n \geq 1 .
$$

Again, the boundary condition (1.2) and initial condition (1.3) are discretized in (2.2) and (2.3), respectively. In addition, the first step can be computed by any 
explicit second or higher order time integrator, e.g., the second-order modified Euler method, as

$$
\begin{gathered}
\psi_{j k}^{1}=\psi_{j k}^{0}-i \tau\left[\left(-\frac{1}{2} \delta_{\nabla}^{2}+V_{j k}-\Omega L_{z}^{h}\right) \psi_{j k}^{(1)}+\beta\left|\psi_{j k}^{(1)}\right|^{2} \psi_{j k}^{(1)}\right], \quad(j, k) \in \mathcal{T}_{M}, \\
\psi_{j k}^{(1)}=\psi_{j k}^{0}-i \frac{\tau}{2}\left[\left(-\frac{1}{2} \delta_{\nabla}^{2}+V_{j k}-\Omega L_{z}^{h}\right) \psi_{j k}^{0}+\beta\left|\psi_{j k}^{0}\right|^{2} \psi_{j k}^{0}\right] .
\end{gathered}
$$

For this SIFD method, at each time step, only a linear system is to be solved, which is much less expensive than that of the CNFD method in practical computation.

2.2. Main error estimate results. Before we state our main error estimate results, we denote the space

$$
\begin{aligned}
X_{M} & =\left\{u=\left(u_{j k}\right)_{(j, k) \in \mathcal{T}_{M}^{0}} \mid u_{0 k}=u_{M k}=u_{j 0}=u_{j K}=0,(j, k) \in \mathcal{T}_{M}^{0}\right\} \\
& \subset \mathbb{C}^{(M+1) \times(K+1)},
\end{aligned}
$$

and define norms and inner product over $X_{M}$ as

$$
\begin{aligned}
& \|u\|_{2}^{2}=\Delta x \Delta y \sum_{j=0}^{M-1} \sum_{k=0}^{K-1}\left|u_{j k}\right|^{2} \\
& \left\|\delta_{\nabla}^{+} u\right\|_{2}^{2}=\Delta x \Delta y \sum_{j=0}^{M-1} \sum_{k=0}^{K-1}\left(\left|\delta_{x}^{+} u_{j k}\right|^{2}+\left|\delta_{y}^{+} u_{j k}\right|^{2}\right), \\
& \|u\|_{\infty}=\sup _{0 \leq j \leq M-1,0 \leq k \leq K-1}\left|u_{j k}\right|, \\
& \|u\|_{p}^{p}=\Delta x \Delta y \sum_{j=0}^{M-1} \sum_{k=0}^{K-1}\left|u_{j k}\right|^{p}, \quad 0<p<\infty, \\
& \text { (2.8) } \mathcal{E}(u)=\frac{1}{2}\left\|\delta_{\nabla}^{+} u\right\|_{2}^{2}+\Delta x \Delta y \sum_{j=1}^{M-1} \sum_{k=1}^{K-1}\left[V_{j k}\left|u_{j k}\right|^{2}-\Omega \bar{u}_{j k} L_{z}^{h} u_{j k}\right], \quad \forall u \in X_{M}, \\
& E_{h}(u)=\frac{1}{2}\left\|\delta_{\nabla}^{+} u\right\|_{2}^{2}+\frac{\beta}{2}\|u\|_{4}^{4}+\Delta x \Delta y \sum_{j=1}^{M-1} \sum_{k=1}^{K-1}\left[V_{j k}\left|u_{j k}\right|^{2}-\Omega \bar{u}_{j k} L_{z}^{h} u_{j k}\right], \\
& (u, v)=\Delta x \Delta y \sum_{j=0}^{M-1} \sum_{k=0}^{K-1} u_{j k} \bar{v}_{j k}, \\
& \langle u, v\rangle=\Delta x \Delta y \sum_{j=1}^{M-1} \sum_{k=1}^{K-1} u_{j k} \bar{v}_{j k}, \quad \forall u, v \in X_{M} .
\end{aligned}
$$

We also make the following assumptions:

(A) Assumption on the trapping potential $V(\mathbf{x})$ and rotation speed $\Omega$, i.e., there exists a constant $\gamma>0$ such that

$$
V(\mathbf{x}) \in C^{1}(U), \quad V(\mathbf{x}) \geq \frac{1}{2} \gamma^{2}\left(x^{2}+y^{2}\right), \quad \forall \mathbf{x} \in U, \quad|\Omega|<\gamma ;
$$


and assumption on the exact solution $\psi$, i.e., let $0<T<T_{\max }$ with $T_{\max }$ be the maximal existing time of the solution [15, 23]:

$$
\begin{gathered}
\psi \in C^{3}\left([0, T] ; W^{1, \infty}(U)\right) \cap C^{2}([0, T] ; \\
\left.W^{3, \infty}(U)\right) \cap C^{0}\left([0, T] ; W^{5, \infty}(U) \cap H_{0}^{1}(U)\right) .
\end{gathered}
$$

Define the "error" function $e^{n} \in X_{M}$ as

$$
e_{j k}^{n}=\psi\left(x_{j}, y_{k}, t_{n}\right)-\psi_{j k}^{n}, \quad(j, k) \in \mathcal{T}_{M}^{0}, \quad n \geq 0 .
$$

Then for the SIFD method, we have

Theorem 2.1 (Convergence of SIFD). Assume $h \lesssim h_{\min }$ and $\tau \lesssim h$, under assumptions $(\mathrm{A})$ and $(\mathrm{B})$, there exist $h_{0}>0$ and $0<\tau_{0}<\frac{1}{4}$ sufficiently small, when $0<h \leq h_{0}$ and $0<\tau \leq \tau_{0}$, we have the following optimal error estimate for the SIFD method (2.4) with (2.2), (2.3) and (2.5)

$$
\left\|e^{n}\right\|_{2} \lesssim h^{2}+\tau^{2}, \quad\left\|\delta_{\nabla}^{+} e^{n}\right\|_{2} \lesssim h^{3 / 2}+\tau^{3 / 2}, \quad 0 \leq n \leq \frac{T}{\tau} .
$$

In addition, if either $\Omega=0$ and $V(\mathbf{x})=0$ or $\psi \in C^{0}\left([0, T] ; H_{0}^{2}(U)\right)$, we have the optimal error estimates:

$$
\left\|e^{n}\right\|_{2}+\left\|\delta_{\nabla}^{+} e^{n}\right\|_{2} \lesssim h^{2}+\tau^{2}, \quad 0 \leq n \leq \frac{T}{\tau} .
$$

Similarly, for the CNFD method, we have

Theorem 2.2 (Convergence of CNFD). Suppose $h \lesssim h_{\min }, \tau \lesssim h$ and either $\beta \geq 0$ or $\beta<0$ with $\left\|\psi^{0}\right\|_{2}^{2}<\frac{1}{|\beta|}\left(1-\frac{\Omega^{2}}{\gamma^{2}}\right)$, under assumption $(\mathrm{A})$, there exists $h_{0}>0$ sufficiently small, when $0<h \leq h_{0}$, the discretization (2.1) with (2.2) and (2.3) admits a unique solution $\psi^{n}\left(0 \leq n \leq \frac{T}{\tau}\right)$. Furthermore, under assumption (B), there exist $h_{0}>0$ and $\tau_{0}>0$ sufficiently small, when $0<h \leq h_{0}$ and $0<\tau \leq \tau_{0}$, we have the following error estimate:

$$
\left\|e^{n}\right\|_{2} \lesssim h^{2}+\tau^{2}, \quad\left\|\delta_{\nabla}^{+} e^{n}\right\|_{2} \lesssim h^{3 / 2}+\tau^{3 / 2}, \quad 0 \leq n \leq \frac{T}{\tau} .
$$

In addition, if either $\Omega=0$ and $V(\mathbf{x})=0$ or $\psi \in C^{0}\left([0, T] ; H_{0}^{2}(U)\right)$, we have the optimal error estimates:

$$
\left\|e^{n}\right\|_{2}+\left\|\delta_{\nabla}^{+} e^{n}\right\|_{2} \lesssim h^{2}+\tau^{2}, \quad 0 \leq n \leq \frac{T}{\tau} .
$$

\section{Error estimates For the SIFD method}

In this section, we establish optimal error estimates for the SIFD method (2.4) with (2.2), (2.3) and (2.5) in $l^{2}$-norm, discrete $H^{1}$-norm and $l^{\infty}$-norm. Let $\psi^{n} \in$ $X_{M}$ be the numerical solution of the SIFD method and $e^{n} \in X_{M}$ the error function.

From (2.8) and (2.10), we have

Lemma 3.1. The following equalities hold:

$$
\begin{aligned}
& \left\langle\delta_{x} u, v\right\rangle=-\left\langle u, \delta_{x} v\right\rangle, \quad\left\langle\delta_{x}^{2} u, v\right\rangle=-\left(\delta_{x}^{+} u, \delta_{x}^{+} v\right), \\
& \left\langle\delta_{y} u, v\right\rangle=-\left\langle u, \delta_{y} v\right\rangle, \quad\left\langle\delta_{y}^{2} u, v\right\rangle=-\left(\delta_{y}^{+} u, \delta_{y}^{+} v\right), \quad \forall u, v \in X_{M}, \\
& \|u\|_{2}^{2} \lesssim\left\|\delta_{\nabla}^{+} u\right\|_{2}^{2}, \quad\|u\|_{4}^{4} \leq\|u\|_{2}^{2} \cdot\left\|\delta_{\nabla}^{+} u\right\|_{2}^{2}, \quad \forall u \in X_{M} .
\end{aligned}
$$


In addition, under assumption (A), we have

$$
\frac{1}{2}\left(1-\frac{\Omega^{2}}{\gamma^{2}}\right)\left\|\delta_{\nabla}^{+} u\right\|_{2}^{2} \leq \mathcal{E}(u) \lesssim\left\|\delta_{\nabla}^{+} u\right\|_{2}^{2}+\|u\|_{2}^{2} \lesssim\left\|\delta_{\nabla}^{+} u\right\|_{2}^{2}, \quad \forall u \in X_{M} .
$$

Proof. The equality (3.1) follows from (2.10) by using summation by parts as

$$
\begin{aligned}
\left\langle\delta_{x} u, v\right\rangle & =\Delta x \Delta y \sum_{j=1}^{M-1} \sum_{k=1}^{K-1} \frac{u_{j+1 k}-u_{j-1 k}}{2 \Delta x} \bar{v}_{j k} \\
& =\Delta x \Delta y \sum_{j=1}^{M-1} \sum_{k=1}^{K-1} u_{j k} \frac{\bar{v}_{j-1 k}-\bar{v}_{j+1 k}}{2 \Delta x}=-\left\langle u, \delta_{x} v\right\rangle, \\
\left\langle\delta_{x}^{2} u, v\right\rangle & =\Delta x \Delta y \sum_{j=1}^{M-1} \sum_{k=1}^{K-1} \frac{u_{j+1 k}-2 u_{j k}+u_{j-1 k}}{(\Delta x)^{2}} \bar{v}_{j k} \\
& =\Delta x \Delta y \sum_{j=0}^{M-1} \sum_{k=0}^{K-1} \frac{u_{j+1 k}-u_{j k}}{\Delta x} \frac{\bar{v}_{j, k}-\bar{v}_{j+1 k}}{\Delta x} \\
& =-\left(\delta_{x}^{+} u, \delta_{x}^{+} v\right), \quad \forall u, v \in X_{M} .
\end{aligned}
$$

Similarly, we can get (3.2). For $u \in X_{M}$, we have

$$
\begin{aligned}
\left|\left(u_{j k}\right)^{2}\right| & =\left|\sum_{l=0}^{j-1}\left[\left(u_{l+1 k}\right)^{2}-\left(u_{l k}\right)^{2}\right]\right|=\Delta x\left|\sum_{l=0}^{j-1}\left[u_{l+1 k}+u_{l k}\right] \delta_{x}^{+} u_{l k}\right| \\
& \leq \Delta x \sum_{l=0}^{j-1}\left|u_{l+1 k}+u_{l k}\right| \cdot\left|\delta_{x}^{+} u_{l k}\right| \\
& \leq \sqrt{2} \Delta x \sqrt{\sum_{l=0}^{M-1}\left|\delta_{x}^{+} u_{l k}\right|^{2}} \sqrt{\sum_{l=0}^{M-1}\left|u_{l k}\right|^{2}}, \quad(j, k) \in \mathcal{T}_{M} .
\end{aligned}
$$

Similarly, we have

$$
\left|\left(u_{j k}\right)^{2}\right| \leq \sqrt{2} \Delta y \sqrt{\sum_{m=0}^{K-1}\left|\delta_{y}^{+} u_{j m}\right|^{2}} \sqrt{\sum_{m=0}^{K-1}\left|u_{j m}\right|^{2}}, \quad(j, k) \in \mathcal{T}_{M} .
$$

Combining (3.5) and (3.6), using the Cauchy inequality, we get

$$
\begin{aligned}
& \|u\|_{4}^{4}=\Delta x \Delta y \sum_{j=0}^{M-1} \sum_{k=0}^{K-1}\left|u_{j k}\right|^{4}=\Delta x \Delta y \sum_{j=0}^{M-1} \sum_{k=0}^{K-1}\left|u_{j k}\right|^{2} \cdot\left|u_{j k}\right|^{2} \\
& \leq 2(\Delta x \Delta y)^{2} \sum_{j=0}^{M-1} \sum_{k=0}^{K-1}\left(\sqrt{\sum_{l=0}^{M-1}\left|\delta_{x}^{+} u_{l k}\right|^{2}} \sqrt{\sum_{l=0}^{M-1}\left|u_{l k}\right|^{2}} \sqrt{\sum_{m=0}^{K-1}\left|\delta_{y}^{+} u_{j m}\right|^{2}} \sqrt{\sum_{m=0}^{K-1}\left|u_{j m}\right|^{2}}\right) \\
& =2(\Delta x \Delta y)^{2} \sum_{k=0}^{K-1}\left(\sqrt{\sum_{l=0}^{M-1}\left|\delta_{x}^{+} u_{l k}\right|^{2}} \sqrt{\sum_{l=0}^{M-1}\left|u_{l k}\right|^{2}}\right) \sum_{j=0}^{M-1}\left(\sqrt{\sum_{m=0}^{K-1}\left|\delta_{y}^{+} u_{j m}\right|^{2}} \sqrt{\sum_{m=0}^{K-1}\left|u_{j m}\right|^{2}}\right) \\
& \leq 2(\Delta x \Delta y)^{2} \sqrt{\sum_{k=0}^{K-1} \sum_{l=0}^{M-1}\left|\delta_{x}^{+} u_{l k}\right|^{2}} \sqrt{\sum_{k=0}^{K-1} \sum_{l=0}^{M-1}\left|u_{l k}\right|^{2}} \sqrt{\sum_{j=0}^{M-1} \sum_{m=0}^{K-1}\left|\delta_{y}^{+} u_{j m}\right|^{2}} \sqrt{\sum_{j=0}^{M-1} \sum_{m=0}^{K-1}\left|u_{j m}\right|^{2}} \\
& \leq\left\|\delta_{\nabla}^{+} u\right\|_{2}^{2} \cdot\|u\|_{2}^{2}, \quad u \in X_{M} .
\end{aligned}
$$


The first inequality in (3.3) can be proved in a similar way. From (2.8) and summation by parts, we get

$$
\begin{aligned}
\sum_{j=1}^{M-1} \sum_{k=1}^{K-1} \bar{u}_{j k} L_{z}^{h} u_{j k} & =-i \sum_{j=1}^{M-1} \sum_{k=1}^{K-1} \bar{u}_{j k}\left(x_{j} \delta_{y} u_{j k}-y_{k} \delta_{x} u_{j k}\right) \\
& =-i \sum_{j=1}^{M-1} \sum_{k=1}^{K-1} u_{j k}\left(x_{j} \delta_{y} \bar{u}_{j k}-y_{k} \delta_{x} \bar{u}_{j k}\right) \\
& =\sum_{j=1}^{M-1} \sum_{k=1}^{K-1} u_{j k} \bar{L}_{z}^{h} \bar{u}_{j k} \quad \in \mathbb{R}, \quad \forall u \in X_{M},
\end{aligned}
$$

which immediately implies that $\mathcal{E}(u) \in \mathbb{R}$ for all $u \in X_{M}$. In addition, using the Cauchy inequality and triangle inequality, noticing assumption (A), we get for $u \in X_{M}$,

$$
\begin{aligned}
& -\Omega \sum_{j=1}^{M-1} \sum_{k=1}^{K-1} \bar{u}_{j k} L_{z}^{h} u_{j k} \\
& \quad=\frac{\Omega}{2} \sum_{j=1}^{M-1} \sum_{k=1}^{K-1} i \bar{u}_{j k}\left[x_{j}\left(\delta_{y}^{+} u_{j k}+\delta_{y}^{+} u_{j, k-1}\right)-y_{k}\left(\delta_{x}^{+} u_{j k}+\delta_{x}^{+} u_{j-1, k}\right)\right] \\
& \quad \geq-\sum_{j=0}^{M-1} \sum_{k=0}^{K-1}\left[V_{j k}\left|u_{j k}\right|^{2}+\frac{\Omega^{2}}{2 \gamma^{2}}\left(\left|\delta_{x}^{+} u_{j k}\right|^{2}+\left|\delta_{y}^{+} u_{j k}\right|^{2}\right)\right]
\end{aligned}
$$

Plugging (3.8) into (2.8) and noticing (2.6), we get (3.4) immediately.

From now on, without loss of generality, we assume that $\Delta x=\Delta y=h$. From (3.4) in Lemma 3.1, we have

Lemma 3.2 (Solvability of the difference equations). Under assumption (A), for any given initial data $\psi^{0} \in X_{M}$, there exists a unique solution $\psi^{n} \in X_{M}$ of (2.5) for $n=1$ and (2.4) for $n>1$.

Proof. The assertion for $n=1$ is obviously true. In SIFD (2.5), for given $\psi^{n-1}, \psi^{n} \in$ $X_{M}(n \geq 1)$, we first prove the uniqueness. Suppose there exist two solutions $\psi^{(1)}, \psi^{(2)} \in X_{M}$ satisfying the SIFD scheme (2.4), i.e., for $(j, k) \in \mathcal{T}_{M}$,

$$
\begin{aligned}
& i \frac{\psi_{j k}^{(1)}-\psi_{j k}^{n-1}}{2 \tau}=\left[-\frac{1}{2} \delta_{\nabla}^{2}+V_{j k}-\Omega L_{z}^{h}\right] \frac{\psi_{j k}^{(1)}+\psi_{j k}^{n-1}}{2}+\beta\left|\psi_{j k}^{n}\right|^{2} \psi_{j k}^{n}, \\
& i \frac{\psi_{j k}^{(2)}-\psi_{j k}^{n-1}}{2 \tau}=\left[-\frac{1}{2} \delta_{\nabla}^{2}+V_{j k}-\Omega L_{z}^{h}\right] \frac{\psi_{j k}^{(2)}+\psi_{j k}^{n-1}}{2}+\beta\left|\psi_{j k}^{n}\right|^{2} \psi_{j k}^{n} .
\end{aligned}
$$

Denote $u=\psi^{(1)}-\psi^{(2)} \in X_{M}$ and subtract (3.10) from (3.9), we have

$$
i \frac{u_{j k}}{\tau}=\left[-\frac{1}{2} \delta_{\nabla}^{2}+V_{j k}-\Omega L_{z}^{h}\right] u_{j k}, \quad(j, k) \in \mathcal{T}_{M}
$$

Multiplying both sides of (3.11) by $\bar{u}_{j k}$ and summing together for $(j, k) \in \mathcal{T}_{M}$, using the summation by parts formula and taking imaginary parts, using (3.4) from Lemma 3.1, we obtain $\|u\|_{2}^{2}=0$, which implies $u=0$. Hence $\psi^{(1)}=\psi^{(2)}$, i.e., the solution of (2.4) is unique. 
Next, we prove the existence. For $(j, k) \in \mathcal{T}_{M}$, rewrite equation (2.4) as

$$
i \psi_{j k}^{n+1}+\tau\left[-\frac{1}{2} \delta_{\nabla}^{2}+V_{j k}-\Omega L_{z}^{h}\right] \psi_{j k}^{n+1}+P_{j k}=0,
$$

where $P \in X_{M}$ is defined as

$$
P_{j k}=-i \psi_{j k}^{n-1}+2 \tau \beta\left|\psi_{j k}^{n}\right|^{2} \psi_{j k}^{n}+\tau\left[-\frac{1}{2} \delta_{\nabla}^{2}+V_{j k}-\Omega L_{z}^{h}\right] \psi_{j k}^{n-1} .
$$

Consider the map $G: \psi^{*} \in X_{M} \rightarrow G\left(\psi^{*}\right) \in X_{M}$ defined as

$$
G\left(\psi^{*}\right)_{j k}=i \psi_{j k}^{*}+\tau\left[-\frac{1}{2} \delta_{\nabla}^{2}+V_{j k}-\Omega L_{z}^{h}\right] \psi_{j k}^{*}+P_{j k}, \quad(j, k) \in \mathcal{T}_{M} .
$$

We know that $G$ is continuous from $X_{M}$ to $X_{M}$. Noticing (3.4) in Lemma 3.1, we have

$$
\operatorname{Im}\left(G\left(\psi^{*}\right), \psi^{*}\right)=\left\|\psi^{*}\right\|_{2}^{2}+\operatorname{Im}\left(P, \psi^{*}\right) \geq\left\|\psi^{*}\right\|_{2}^{2}-\|P\|_{2}\left\|\psi^{*}\right\|_{2},
$$

which immediately implies

$$
\lim _{\left\|\psi^{*}\right\|_{2} \rightarrow \infty} \frac{\left|\left(G\left(\psi^{*}\right), \psi^{*}\right)\right|}{\left\|\psi^{*}\right\|_{2}}=\infty .
$$

Hence $G: X_{M} \rightarrow X_{M}$ is surjective [27] and there exists a solution $\psi^{n+1} \in X_{M}$ satisfying $G\left(\psi^{n+1}\right)=0$. Then $\psi^{n+1}$ satisfies the equation (2.4). The proof is complete.

Define the local truncation error $\eta^{n} \in X_{M}$ of the SIFD method (2.4) with (2.2), (2.3) and (2.5) for $n \geq 1$ as

$$
\begin{aligned}
\eta_{j k}^{n}:= & i \delta_{t} \psi\left(x_{j}, y_{k}, t_{n}\right)-\left[-\frac{1}{2} \delta_{\nabla}^{2}-\Omega L_{z}^{h}+V_{j k}\right] \frac{\psi\left(x_{j}, y_{k}, t_{n-1}\right)+\psi\left(x_{j}, y_{k}, t_{n+1}\right)}{2} \\
& -\beta\left|\psi\left(x_{j}, y_{k}, t_{n}\right)\right|^{2} \psi\left(x_{j}, y_{k}, t_{n}\right), \quad(j, k) \in \mathcal{T}_{M},
\end{aligned}
$$

and by noticing (2.3) for $n=0$ as

$$
\begin{aligned}
& \eta_{j k}^{0}:=i \delta_{t}^{+} \psi\left(x_{j}, y_{k}, 0\right)-\left(-\frac{1}{2} \delta_{\nabla}^{2}+V_{j k}-\Omega L_{z}^{h}\right) \psi_{j k}^{(1)} \\
&-\beta\left|\psi_{j k}^{(1)}\right|^{2} \psi_{j k}^{(1)}, \quad(j, k) \in \mathcal{T}_{M}, \\
& \psi_{j k}^{(1)}=\psi_{0}\left(x_{j}, y_{k}\right)-i \frac{\tau}{2}\left[\left(-\frac{1}{2} \delta_{\nabla}^{2}+V_{j k}-\Omega L_{z}^{h}\right) \psi_{0}\left(x_{j}, y_{k}\right)\right. \\
&\left.+\beta\left|\psi_{0}\left(x_{j}, y_{k}\right)\right|^{2} \psi_{0}\left(x_{j}, y_{k}\right)\right] .
\end{aligned}
$$

Then we have

Lemma 3.3 (Local truncation error). Assuming $V(\mathbf{x}) \in C(\bar{U})$, under assumption (B), we have

$$
\left\|\eta^{n}\right\|_{\infty} \lesssim \tau^{2}+h^{2}, \quad 0 \leq n \leq \frac{T}{\tau}-1, \quad \text { and } \quad\left\|\delta_{\nabla}^{+} \eta^{0}\right\|_{\infty} \lesssim \tau+h .
$$

In addition, assuming $V(\mathbf{x}) \in C^{1}(\bar{U})$ and $\tau \lesssim h$, we have for $1 \leq n \leq \frac{T}{\tau}-1$,

$$
\left|\delta_{\nabla}^{+} \eta_{j k}^{n}\right| \lesssim \begin{cases}\tau^{2}+h^{2}, & 1 \leq j \leq M-2, \quad 1 \leq k \leq K-2 \\ \tau+h, & j=0, M-1, \text { or } k=0, K-1\end{cases}
$$


Furthermore, assuming either $\Omega=0$ and $V(\mathbf{x})=0$ or $u \in C\left([0, T] ; H_{0}^{2}(U)\right)$, we have

$$
\left\|\delta_{\nabla}^{+} \eta^{n}\right\|_{\infty} \lesssim \tau^{2}+h^{2}, \quad 1 \leq n \leq \frac{T}{\tau}-1
$$

Proof. First, we prove (3.19) and (3.21) when $n=0$. Rewriting $\psi_{j k}^{(1)}$ and then using Taylor's expansion at $\left(x_{j}, y_{k}, 0\right)$, noticing (1.1) and (1.3), we get

$$
\begin{aligned}
& \psi_{j k}^{(1)}=\psi\left(x_{j}, y_{k}, \frac{\tau}{2}\right)+i \frac{\tau}{2}\left[\left(\frac{1}{2} \delta_{\nabla}^{2}-V_{j k}+\Omega L_{z}^{h}\right) \psi_{0}\left(x_{j}, y_{k}\right)\right. \\
& \left.-\beta\left|\psi_{0}\left(x_{j}, y_{k}\right)\right|^{2} \psi_{0}\left(x_{j}, y_{k}\right)+i \frac{\psi\left(x_{j}, y_{k}, \frac{\tau}{2}\right)-\psi_{0}\left(x_{j}, y_{k}\right)}{\tau / 2}\right] \\
& =\psi\left(x_{j}, y_{k}, \frac{\tau}{2}\right)+i \frac{\tau}{2}\left[\frac { h } { 6 } \left[\partial_{x x x} \psi_{0}\left(x_{j}+h \theta_{j k}^{(2)}, y_{k}\right)+\partial_{y y y} \psi_{0}\left(x_{j}, y_{k}+h \theta_{j k}^{(3)}\right)\right.\right. \\
& \left.-3 i \Omega\left(x_{j} \partial_{y y} \psi_{0}\left(x_{j}, y_{k}+h \theta_{j k}^{(4)}\right)-y_{k} \partial_{x x} \psi_{0}\left(x_{j}+h \theta_{j k}^{(5)}, y_{k}\right)\right)\right] \\
& \left.+i \frac{\tau}{4} \partial_{t t} \psi\left(x_{j}, y_{k}, \tau \theta_{j k}^{(1)}\right)\right]=\psi\left(x_{j}, y_{k}, \frac{\tau}{2}\right)+O\left(\tau^{2}+\tau h\right), \quad(j, k) \in \mathcal{T}_{M},
\end{aligned}
$$

where $\theta_{j k}^{(1)} \in[0,1 / 2]$ and $\theta_{j k}^{(2)}, \theta_{j k}^{(3)}, \theta_{j k}^{(4)}, \theta_{j k}^{(5)} \in[-1,1]$ are constants. Similarly, using Taylor's expansion at $\left(x_{j}, y_{k}, \tau / 2\right)$ in (3.18), noticing (1.1) and (3.22), using triangle inequality and assumption $(\mathrm{B})$, we get

$$
\begin{aligned}
& \left|\eta_{j k}^{0}\right| \lesssim \tau^{2}\left\|\partial_{t t t} \psi\right\|_{L^{\infty}}+h^{2}\left[\left\|\partial_{x x x x} \psi\right\|_{L^{\infty}}+\left\|\partial_{y y y y} \psi\right\|_{L^{\infty}}+\left\|\partial_{x x x} \psi\right\|_{L^{\infty}}+\left\|\partial_{y y y} \psi\right\|_{L^{\infty}}\right] \\
& \quad+\tau^{2}\left[\left\|\partial_{t t x x} \psi\right\|_{L^{\infty}}+\left\|\partial_{t t y y} \psi\right\|_{L^{\infty}}+\left\|\partial_{t t x} \psi\right\|_{L^{\infty}}+\left\|\partial_{t t y} \psi\right\|_{L^{\infty}}+\left\|\partial_{t t} \psi\right\|_{L^{\infty}}\|\psi\|_{L^{\infty}}^{2}\right] \\
& \quad+\tau h\left[\left\|\psi_{0}\right\|_{W^{5, \infty}(U)}+\|\psi\|_{L^{\infty}}^{2}\left\|\psi_{0}\right\|_{W^{3, \infty}(U)}\right]+O\left(h^{4}+\tau^{4}\right) \\
& \quad \lesssim \tau^{2}+h^{2}, \quad(j, k) \in \mathcal{T}_{M},
\end{aligned}
$$

where the $L^{\infty}$-norm means $\|f\|_{L^{\infty}}:=\sup _{0 \leq t \leq T} \sup _{\mathbf{x} \in U}|f(\mathbf{x}, t)|$. This immediately implies (3.19) when $n=0$ as

$$
\left\|\eta^{0}\right\|_{\infty}=\max _{(j, k) \in \mathcal{T}_{M}^{0}}\left|\eta_{j k}^{0}\right| \lesssim \tau^{2}+h^{2} .
$$

Similarly, noticing $\tau \lesssim h$,

$$
\left|\delta_{\nabla}^{+} \eta_{j k}^{0}\right| \lesssim \frac{1}{h}\left|\eta_{j k}^{0}\right| \lesssim \tau+h, \quad(j, k) \in \mathcal{T}_{M},
$$

which immediately implies (3.21) when $n=0$. Now we prove (3.19), (3.20) and (3.21) when $n \geq 1$. Using Taylor's expansion at $\left(x_{j}, y_{k}, t_{n}\right)$ in (3.17), noticing (1.1), using triangle inequality and assumption (B), we have

$$
\begin{aligned}
\left|\eta_{j k}^{n}\right| \lesssim & h^{2}\left[\left\|\partial_{x x x x} \psi\right\|_{L^{\infty}}+\left\|\partial_{y y y y} \psi\right\|_{L^{\infty}}+\left\|\partial_{y y y} \psi\right\|_{L^{\infty}}+\left\|\partial_{x x x} \psi\right\|_{L^{\infty}}\right] \\
& +\tau^{2}\left[\left\|\partial_{t t t} \psi\right\|_{L^{\infty}}+\left\|\partial_{t t x x} \psi\right\|_{L^{\infty}}+\left\|\partial_{t t y y} \psi\right\|_{L^{\infty}}+\left\|\partial_{y t t} \psi\right\|_{L^{\infty}}+\left\|\partial_{x t t} \psi\right\|_{L^{\infty}}\right] \\
\lesssim & \tau^{2}+h^{2}, \quad(j, k) \in \mathcal{T}_{M}, \quad 1 \leq n \leq \frac{T}{\tau}-1
\end{aligned}
$$


which implies (3.19) for $n \geq 1$ and (3.20) for $j=0, M-1$ or $k=0, K-1$. Similarly, we have

$$
\begin{aligned}
\left|\delta_{\nabla}^{+} \eta_{j k}^{n}\right| \lesssim & h^{2}\left[\left\|\partial_{x x x x} \nabla \psi\right\|_{L^{\infty}}+\left\|\partial_{y y y y} \nabla \psi\right\|_{L^{\infty}}+\left\|\partial_{y y y} \nabla \psi\right\|_{L^{\infty}}+\left\|\partial_{x x x} \nabla \psi\right\|_{L^{\infty}}\right] \\
& +\tau^{2}\left[\left\|\partial_{t t t} \nabla \psi\right\|_{L^{\infty}}+\left\|\partial_{t t x x} \nabla \psi\right\|_{L^{\infty}}+\left\|\partial_{t t y y} \nabla \psi\right\|_{L^{\infty}}\right. \\
& \left.+\left\|\partial_{y t t} \nabla \psi\right\|_{L^{\infty}}+\left\|\partial_{x t t} \nabla \psi\right\|_{L^{\infty}}\right] \\
\lesssim & \tau^{2}+h^{2}, \quad 1 \leq j \leq M-2,1 \leq k \leq K-2, \quad 1 \leq n \leq \frac{T}{\tau}-1,
\end{aligned}
$$

which immediately implies (3.20) for $n \geq 1$. In addition, if $\Omega=0$ and $V(\mathbf{x})=0$, using equation (1.1), we obtain the following derivatives of $\psi$ on the boundary are 0, i.e.,

$$
\left.\partial_{x x} \psi\right|_{\partial U}=\left.\partial_{y y} \psi\right|_{\partial U}=\left.\partial_{x x x x} \psi\right|_{\partial U}=\left.\partial_{y y y y} \psi\right|_{\partial U}=0 .
$$

Hence (3.23) holds for the boundary case, i.e., $j=0, M-1$ or $k=0, K-1$, and we could obtain (3.21) for $n \geq 1$. If $\psi \in C^{0}\left([0, T] ; H_{0}^{2}(U)\right)$, using the equation (1.1), we obtain that

$$
\left.\partial_{x}^{m} \partial_{y}^{n} \psi\right|_{\partial U}=0, \quad m \geq 0, n \geq 0, m+n \leq 4,
$$

and similarly (3.23) holds for $j=0, M-1$ or $k=0, K-1$, then we could obtain (3.21) for $n \geq 1$. Thus, the proof is complete.

Theorem 3.1 ( $l^{2}$-norm estimate). Assume $\tau \lesssim h$, under assumptions (A) and (B), there exist $h_{0}>0$ and $0<\tau_{0}<\frac{1}{4}$ sufficiently small, when $0<h \leq h_{0}$ and $0<\tau \leq \tau_{0}$, we have

$$
\left\|e^{n}\right\|_{2} \lesssim \tau^{2}+h^{2}, \quad\left\|\psi^{n}\right\|_{\infty} \leq 1+M_{1}, \quad 0 \leq n \leq \frac{T}{\tau},
$$

where $M_{1}=\max _{0 \leq t \leq T}\|\psi(\cdot, t)\|_{L^{\infty}(U)}$.

Proof. We will prove this theorem by the method of mathematical induction. From (1.3) and (2.3), it is straightforward to see that (3.26) is valid when $n=0$. From (2.5) and (3.18), noticing (3.19), we get

$$
\begin{aligned}
\left|e_{j k}^{1}\right| & =\left|\psi\left(x_{j}, y_{k}, t_{1}\right)-\psi_{j k}^{1}\right|=\left|-i \tau \eta_{j k}^{0}\right| \\
& \lesssim \tau\left(\tau^{2}+h^{2}\right) \lesssim \tau^{2}+h^{2}, \quad(j, k) \in \mathcal{T}_{M},
\end{aligned}
$$

which immediately implies the first inequality in (3.26) when $n=1$. This, together with the triangle inequality, when $\tau$ and $h$ are sufficiently small, we obtain

$$
\left|\psi_{j k}^{1}\right| \leq\left|\psi\left(x_{j}, y_{k}, t_{1}\right)\right|+\left|e_{j k}^{1}\right| \leq M_{1}+C\left(\tau^{2}+h^{2}\right) \leq 1+M_{1}, \quad(j, k) \in \mathcal{T}_{M},
$$

which immediately implies the second inequality in (3.26) when $n=1$. Now we assume that (3.26) is valid for all $0 \leq n \leq m-1 \leq \frac{T}{\tau}-1$, then we need to show that it is still valid when $n=m$. In order to do so, subtracting (3.17) from (2.4), noticing (1.2) and (2.2), we obtain the following equation for the "error" function $e^{n} \in X_{M}$ :

$$
i \delta_{t} e_{j k}^{n}=\left[-\frac{1}{2} \delta_{\nabla}^{2}+V_{j k}-\Omega L_{z}^{h}\right] \frac{e_{j k}^{n+1}+e_{j k}^{n-1}}{2}+\xi_{j k}^{n}+\eta_{j k}^{n}, \quad(j, k) \in \mathcal{T}_{M}, \quad n \geq 1,
$$


where $\xi^{n} \in X_{M}(n \geq 1)$ is defined as

$$
\begin{aligned}
\xi_{j k}^{n}= & \beta\left|\psi\left(x_{j}, y_{k}, t_{n}\right)\right|^{2} \psi\left(x_{j}, y_{k}, t_{n}\right)-\beta\left|\psi_{j k}^{n}\right|^{2} \psi_{j k}^{n} \\
= & \beta\left|\psi\left(x_{j}, y_{k}, t_{n}\right)\right|^{2} e_{j k}^{n}+\beta\left(\overline{e_{j k}^{n}} \psi_{j k}^{n}\right. \\
& \left.+\overline{\psi\left(x_{j}, y_{k}, t_{n}\right)} e_{j k}^{n}\right) \psi_{j k}^{n}, \quad(j, k) \in \mathcal{T}_{M} .
\end{aligned}
$$

Noticing (3.26), we have the following estimate,

$\left\|\xi^{n}\right\|_{2}^{2} \leq 9 \beta^{2}\left(1+M_{1}\right)^{4}\left\|e^{n}\right\|_{2}^{2}, \quad\left\|\delta_{\nabla}^{+} \xi^{n}\right\|_{2}^{2} \lesssim\left\|\delta_{\nabla}^{+} e^{n}\right\|_{2}^{2}+\left\|e^{n}\right\|_{2}^{2}, \quad 1 \leq n \leq m-1$.

Multiplying both sides of (3.28) by $\overline{e_{j k}^{n+1}+e_{j k}^{n-1}}$ and summing all together for $(j, k) \in \mathcal{T}_{M}$, taking imaginary parts, using the triangular and Cauchy inequalities, noticing (3.19) and (3.30), we have for $1 \leq n \leq m-1$,

$$
\begin{aligned}
\| e^{n+1} & \left\|_{2}^{2}-\right\| e^{n-1} \|_{2}^{2} \\
& =2 \tau \operatorname{Im}\left(\xi^{n}+\eta^{n}, e^{n+1}+e^{n-1}\right) \\
& \leq 2 \tau\left[\left\|e^{n+1}\right\|_{2}^{2}+\left\|e^{n-1}\right\|_{2}^{2}+\left\|\eta^{n}\right\|_{2}^{2}+\left\|\xi^{n}\right\|_{2}^{2}\right] \\
& \leq C \tau\left(h^{2}+\tau^{2}\right)^{2}+2 \tau\left(\left\|e^{n+1}\right\|_{2}^{2}+\left\|e^{n-1}\right\|_{2}^{2}\right)+18 \tau \beta^{2}\left(1+M_{1}\right)^{4}\left\|e^{n}\right\|_{2}^{2} .
\end{aligned}
$$

When $\tau \leq \frac{1}{4}$, we have

$$
\left\|e^{n+1}\right\|_{2}^{2}-\left\|e^{n-1}\right\|_{2}^{2} \leq C \tau\left[\left(h^{2}+\tau^{2}\right)^{2}+\left\|e^{n-1}\right\|_{2}^{2}+\beta^{2}\left(1+M_{1}\right)^{4}\left\|e^{n}\right\|_{2}^{2}\right] .
$$

Summing the above inequality for $n=1,2, \ldots, m-1$, we get

$$
\left\|e^{m}\right\|_{2}^{2}+\left\|e^{m-1}\right\|_{2}^{2} \leq C T\left(h^{2}+\tau^{2}\right)^{2}+C \tau\left[1+\beta^{2}\left(M_{1}+1\right)^{4}\right] \sum_{l=1}^{m-1}\left\|e^{l}\right\|_{2}^{2}, \quad 1 \leq m \leq \frac{T}{\tau} .
$$

Using the discrete Gronwall inequality [16, 21, 28, and noticing $\left\|e^{0}\right\|_{2}=0$ and $\left\|e^{1}\right\|_{2} \lesssim h^{2}+\tau^{2}$, we immediately obtain the first inequality in (3.26) for $n=m$. Using the inverse inequality, triangle inequality and $l^{2}$-norm estimate, noticing $\tau \lesssim h$, we obtain

$$
\begin{aligned}
\left|\psi_{j k}^{m}\right| & \leq\left|\psi\left(x_{j}, y_{k}, t_{m}\right)\right|+\left|e_{j k}^{m}\right| \leq M_{1}+\left\|e^{m}\right\|_{\infty} \leq M_{1}+\frac{C}{h}\left\|e^{m}\right\|_{2} \\
& \leq M_{1}+\frac{C}{h}\left(h^{2}+\tau^{2}\right) \leq M_{1}+C h, \quad(j, k) \in \mathcal{T}_{M}^{0} .
\end{aligned}
$$

Thus there exists a constant $h_{0}>0$ sufficiently small, when $0<h \leq h_{0}$ and $0<\tau \lesssim h$, we have

$$
\left\|\psi^{m}\right\|_{\infty} \leq 1+M_{1}, \quad 1 \leq m \leq \frac{T}{\tau}
$$

which is the second inequality in (3.26) when $n=m$. Therefore the proof of the theorem is completed by the method of mathematical induction.

Combining Theorem 3.1 and Lemmas 3.1, 3.2 and 3.3, we are now ready to prove the main Theorem 2.1

Proof of Theorem 2.1. We first prove the optimal discrete semi- $H^{1}$ norm convergence rate in the case of either $\Omega=0$ and $V(\mathbf{x})=0$ or $\psi \in C^{0}\left([0, T] ; H_{0}^{2}(U)\right)$. From 
(2.3), we know $e^{0}=0$ and thus (2.12) is valid for $n=0$. From (2.5) and (3.18), noticing (3.19), we get

$$
\begin{aligned}
\left|\delta_{\nabla}^{+} e_{j k}^{1}\right| & =\left|\delta_{\nabla}^{+}\left(\psi\left(x_{j}, y_{k}, t_{1}\right)-\psi_{j k}^{1}\right)\right|=\left|-i \tau \delta_{\nabla}^{+} \eta_{j k}^{0}\right| \\
& \lesssim \tau(\tau+h) \lesssim \tau^{2}+h^{2}, \quad(j, k) \in \mathcal{T}_{M},
\end{aligned}
$$

which immediately implies (2.12) when $n=1$. Multiplying both sides of (3.28) by $\overline{e_{j k}^{n+1}-e_{j k}^{n-1}}$, summing over index $(j, k) \in \mathcal{T}_{M}$ and summation by parts, taking real part and noticing (2.7), we have

$$
\mathcal{E}\left(e^{n+1}\right)-\mathcal{E}\left(e^{n-1}\right)=-2 \operatorname{Re}\left\langle\xi^{n}+\eta^{n}, e^{n+1}-e^{n-1}\right\rangle, \quad n \geq 1,
$$

where $\operatorname{Re}(f)$ denotes the real part of $f$. Rewriting (3.28) as

$$
e_{j k}^{n+1}-e_{j k}^{n-1}=-2 i \tau\left[\xi_{j k}^{n}+\eta_{j k}^{n}+\chi_{j k}^{n}\right], \quad(j, k) \in \mathcal{T}_{M},
$$

where $\chi^{n} \in X_{M}$ is defined as

$$
\chi_{j k}^{n}=\left[-\frac{1}{2} \delta_{\nabla}^{2}+V_{j k}-\Omega L_{h}^{z}\right] \frac{e_{j k}^{n+1}+e_{j k}^{n-1}}{2}, \quad(j, k) \in \mathcal{T}_{M},
$$

then plugging (3.34) into (3.33), we obtain

$$
\begin{aligned}
\mathcal{E}\left(e^{n+1}\right)-\mathcal{E}\left(e^{n-1}\right) & =-4 \tau \operatorname{Im}\left\langle\xi^{n}+\eta^{n}, \xi^{n}+\eta^{n}+\chi^{n}\right\rangle \\
& =-4 \tau \operatorname{Im}\left\langle\xi^{n}+\eta^{n}, \chi^{n}\right\rangle, \quad n \geq 1 .
\end{aligned}
$$

From (3.35) and (3.29), noticing (3.1), (3.2) and (3.4), we have

$$
\begin{aligned}
\left|\left\langle\xi^{n}, \chi^{n}\right\rangle\right|= & \frac{1}{2}\left|\left\langle\xi^{n},\left(-\frac{1}{2} \delta_{\nabla}^{2}+V-\Omega L_{z}^{h}\right)\left(e^{n+1}+e^{n-1}\right)\right\rangle\right| \\
\lesssim & \left|\left\langle\delta_{\nabla}^{+} \xi^{n}, \delta_{\nabla}^{+}\left(e^{n+1}+e^{n-1}\right)\right\rangle\right|+\left|\left\langle\xi^{n}, V\left(e^{n+1}+e^{n-1}\right)\right\rangle\right| \\
& +\left|\left\langle\xi^{n}, \Omega L_{z}^{h}\left(e^{n+1}+e^{n-1}\right)\right\rangle\right| \\
\lesssim & \left\|\delta_{\nabla}^{+} e^{n+1}\right\|_{2}^{2}+\left\|\delta_{\nabla}^{+} e^{n}\right\|_{2}^{2}+\left\|\delta_{\nabla}^{+} e^{n-1}\right\|_{2}^{2}+\left\|e^{n+1}\right\|_{2}^{2}+\left\|e^{n}\right\|_{2}^{2}+\left\|e^{n-1}\right\|_{2}^{2} \\
& +\left\|\delta_{\nabla}^{+} \xi^{n}\right\|_{2}^{2}+\left\|\xi^{n}\right\|_{2}^{2} \\
\lesssim & \left\|\delta_{\nabla}^{+} e^{n+1}\right\|_{2}^{2}+\left\|\delta_{\nabla}^{+} e^{n}\right\|_{2}^{2}+\left\|\delta_{\nabla}^{+} e^{n-1}\right\|_{2}^{2}, \quad 1 \leq n \leq \frac{T}{\tau}-1 .
\end{aligned}
$$

Similarly, noticing (3.30), (3.19) and (3.21), we have

$$
\begin{aligned}
\left|\left\langle\eta^{n}, \chi^{n}\right\rangle\right| & =\frac{1}{2}\left|\left\langle\eta^{n},\left(-\frac{1}{2} \delta_{\nabla}^{2}+V-\Omega L_{z}^{h}\right)\left(e^{n+1}+e^{n-1}\right)\right\rangle\right| \\
& \lesssim\left|\left\langle\delta_{\nabla}^{+} \eta^{n}, \delta_{\nabla}^{+}\left(e^{n+1}+e^{n-1}\right)\right\rangle\right|+\left|\left\langle\eta^{n}, V\left(e^{n+1}+e^{n-1}\right)\right\rangle\right| \\
& +\left|\left\langle\eta^{n}, \Omega L_{z}^{h}\left(e^{n+1}+e^{n-1}\right)\right\rangle\right| \\
& \lesssim\left\|\delta_{\nabla}^{+} e^{n+1}\right\|_{2}^{2}+\left\|\delta_{\nabla}^{+} e^{n}\right\|_{2}^{2}+\left\|\delta_{\nabla}^{+} e^{n-1}\right\|_{2}^{2}+\left\|e^{n+1}\right\|_{2}^{2}+\left\|e^{n}\right\|_{2}^{2}+\left\|e^{n-1}\right\|_{2}^{2} \\
& +\left\|\delta_{\nabla}^{+} \eta^{n+1}\right\|_{2}^{2}+\left\|\eta^{n}\right\|_{2}^{2} \\
& \lesssim\left\|\delta_{\nabla}^{+} e^{n+1}\right\|_{2}^{2}+\left\|\delta_{\nabla}^{+} e^{n}\right\|_{2}^{2}+\left\|\delta_{\nabla}^{+} e^{n-1}\right\|_{2}^{2} \\
& +\left(\tau^{2}+h^{2}\right)^{2}, \quad 1 \leq n \leq \frac{T}{\tau}-1 .
\end{aligned}
$$


Plugging (3.37) and (3.38) into (3.36), using (3.4) and the triangle inequality, we get

$$
\begin{aligned}
\mathcal{E}\left(e^{n+1}\right)-\mathcal{E}\left(e^{n-1}\right) & \lesssim \tau\left(\tau^{2}+h^{2}\right)^{2}+\tau\left[\left\|\delta_{\nabla}^{+} e^{n+1}\right\|_{2}^{2}+\left\|\delta_{\nabla}^{+} e^{n}\right\|_{2}^{2}+\left\|\delta_{\nabla}^{+} e^{n-1}\right\|_{2}^{2}\right] \\
& \lesssim \tau\left(\tau^{2}+h^{2}\right)^{2}+\tau\left[\mathcal{E}\left(e^{n+1}\right)+\mathcal{E}\left(e^{n}\right)+\mathcal{E}\left(e^{n-1}\right)\right], 1 \leq n \leq \frac{T}{\tau}-1 .
\end{aligned}
$$

There exists $\tau_{0}>0$ sufficiently small, when $0<\tau \leq \tau_{0}$, we have

$$
\mathcal{E}\left(e^{n+1}\right)-\mathcal{E}\left(e^{n-1}\right) \lesssim \tau\left(\tau^{2}+h^{2}\right)^{2}+\tau\left[\mathcal{E}\left(e^{n}\right)+\mathcal{E}\left(e^{n-1}\right)\right], \quad 1 \leq n \leq \frac{T}{\tau}-1
$$

Summing the above inequality for $1 \leq n \leq m-1 \leq \frac{T}{\tau}-1$, we get

$$
\mathcal{E}\left(e^{m}\right)+\mathcal{E}\left(e^{m-1}\right) \lesssim T\left(\tau^{2}+h^{2}\right)^{2}+\mathcal{E}\left(e^{1}\right)+\mathcal{E}\left(e^{0}\right)+\tau \sum_{l=1}^{m-1} \mathcal{E}\left(e^{l}\right), \quad 1 \leq m \leq \frac{T}{\tau} .
$$

Using the discrete Gronwall inequality [28, noticing (3.26) and (3.32), we have

$$
\begin{aligned}
\left\|\delta_{\nabla}^{+} e^{m}\right\|_{2}^{2} & \lesssim \mathcal{E}\left(e^{m}\right) \leq \mathcal{E}\left(e^{m}\right)+\mathcal{E}\left(e^{m-1}\right) \lesssim\left(\tau^{2}+h^{2}\right)^{2}+\mathcal{E}\left(e^{1}\right)+\mathcal{E}\left(e^{0}\right) \\
& \lesssim\left(\tau^{2}+h^{2}\right)^{2}+\left\|e^{1}\right\|_{2}^{2}+\left\|\delta_{\nabla}^{+} e^{1}\right\|_{2}^{2} \lesssim\left(\tau^{2}+h^{2}\right)^{2}, \quad 1 \leq m \leq \frac{T}{\tau} .
\end{aligned}
$$

This together with (3.26) imply (2.12). For the case of assumptions (A) and (B) without further assumptions, we will lose half-order convergence rate in the semi$H^{1}$-norm because of the boundary (3.20). Notice that the reminder term is $O\left(h^{2}+\right.$ $\left.\tau^{2}\right)^{3 / 2}$ instead of $O\left(h^{2}+\tau^{2}\right)$ in (3.38), and the the remaining proof is the same. Hence, we will have the $3 / 2$ order convergence rate for discrete semi- $H^{1}$-norm. The proof is complete.

Similarly, as in the proof of Theorem 2.1, we can get error estimate for the mass and energy in the discretized level as

Lemma 3.4 (Estimates on mass and energy). Under the same conditions of Theorem 2.1 with assumptions (A) and (B), we have for $0 \leq n \leq \frac{T}{\tau}$,

$$
\begin{aligned}
\left|\left\|\psi^{n}\right\|_{2}^{2}-N\left(\psi_{0}\right)\right| & =\left|\left\|\psi^{n}\right\|_{2}^{2}-N\left(\psi\left(\cdot, t_{n}\right)\right)\right| \\
& \leq\left|\left\|\psi^{n}\right\|_{2}^{2}-\left\|\Pi_{h} \psi\left(t_{n}\right)\right\|_{2}^{2}\right|+\left|\left\|\Pi_{h} \psi\left(t_{n}\right)\right\|_{2}^{2}-N\left(\psi\left(\cdot, t_{n}\right)\right)\right| \\
& \lesssim h^{3 / 2}+\tau^{3 / 2}, \\
\left|E_{h}\left(\psi^{n}\right)-E\left(\psi_{0}\right)\right| & =\left|E_{h}\left(\psi^{n}\right)-E\left(\psi\left(\cdot, t_{n}\right)\right)\right| \\
& \leq\left|E_{h}\left(\psi^{n}\right)-E_{h}\left(\Pi \psi\left(t_{n}\right)\right)\right|+\left|E_{h}\left(\Pi \psi\left(t_{n}\right)\right)-E\left(\psi\left(\cdot, t_{n}\right)\right)\right| \\
& \lesssim h^{3 / 2}+\tau^{3 / 2},
\end{aligned}
$$

where $\Pi_{h}: X:=\left\{f \in C(\bar{U})|f|_{\partial U}=0\right\} \rightarrow X_{M}$ is the standard project operator defined as

$$
\left(\Pi_{h} f\right)_{j k}=f\left(x_{j}, y_{k}\right), \quad f \in X, \quad\left(\Pi_{h} \psi\left(t_{n}\right)\right)_{j k}=\psi\left(x_{j}, y_{k}, t_{n}\right), \quad(j, k) \in \mathcal{T}_{M}^{0} .
$$

In addition, assume either $\Omega=0$ and $V(\mathbf{x})=0$ or $\psi \in C\left([0, T] ; H_{0}^{2}(U)\right)$, then we have

$$
\left|\left\|\psi^{n}\right\|_{2}^{2}-N\left(\psi_{0}\right)\right|+\left|E_{h}\left(\psi^{n}\right)-E\left(\psi_{0}\right)\right| \lesssim h^{2}+\tau^{2}, \quad 0 \leq n \leq \frac{T}{\tau} .
$$

In addition, from Theorem 2.1 and using the inverse inequality [40, we get immediately the error estimate in $l^{\infty}$-norm for the SIFD method as 
Lemma 3.5 ( $l^{\infty}$-norm estimate). Under the same conditions of Theorem 2.1 with assumptions (A) and (B) and assume $h<1$, we have the following error estimate for the SIFD

$$
\left\|e^{n}\right\|_{\infty} \lesssim \begin{cases}\left(h^{3 / 2}+\tau^{3 / 2}\right)|\ln (h)|, & d=2, \\ h+\tau, & d=3 .\end{cases}
$$

In addition, if either $\Omega=0$ and $V(\mathbf{x})=0$ or $\psi \in C^{0}\left([0, T] ; H_{0}^{2}(U)\right)$, we have

$$
\left\|e^{n}\right\|_{\infty} \lesssim \begin{cases}\left(h^{2}+\tau^{2}\right)|\ln (h)|, & d=2, \\ h^{3 / 2}+\tau^{3 / 2}, & d=3 .\end{cases}
$$

Remark 3.1. If the cubic nonlinear term $\beta|\psi|^{2} \psi$ in (1.1) is replaced by a general nonlinearity $f\left(|\psi|^{2}\right) \psi$, the numerical discretization SIFD and its error estimates in $l^{2}$-norm, $l^{\infty}$-norm and discrete $H^{1}$-norm are still valid provided that the nonlinear real-valued function $f(\rho) \in C^{2}([0, \infty))$.

\section{ERror EStimates FOR the CNFD MEthod}

In this section, we prove optimal error estimate for the CNFD method (2.1) with (2.2) and (2.3) in $l^{2}$-norm, discrete $H^{1}$-norm and $l^{\infty}$-norm. Let $\psi^{n} \in X_{M}$ be the numerical solution of the CNFD method and $e^{n} \in X_{M}$ be the error function.

Lemma 4.1 (Conservation of mass and energy). For the CNFD scheme (2.1) with (2.2) and (2.3), for any mesh size $h>0$, time step $\tau>0$ and initial data $\psi_{0}$, it conserves the mass and energy in the discretized level, i.e.,

$$
\left\|\psi^{n}\right\|_{2}^{2} \equiv\left\|\psi^{0}\right\|_{2}^{2}, \quad E_{h}\left(\psi^{n}\right) \equiv E_{h}\left(\psi^{0}\right), \quad n=0,1,2, \ldots .
$$

Proof. Follow the analogous arguments of the CNFD method for the NLS [16, 21] and we omit the details here for brevity.

Lemma 4.2 (Solvability of the difference equations). For any given $\psi^{n}$, there exists a solution $\psi^{n+1}$ of the CNFD discretization (2.1) with (2.2) and (2.3). In addition, assume $\tau \lesssim h$ and either $\beta \geq 0$ or $\beta<0$ with $\left\|\psi^{0}\right\|_{2}^{2}<\frac{1}{|\beta|}\left(1-\frac{\Omega^{2}}{\gamma^{2}}\right)$, under assumption $(\mathrm{A})$, there exists $h_{0}>0$ sufficiently small, when $0<h \leq h_{0}$, the solution is unique.

Proof. First, we prove the existence of a solution of the CNFD discretization (2.1). In order to do so, for any given $\psi^{n} \in X_{M}$, we rewrite the equation (2.1) as

$$
\psi^{n+1 / 2}=\psi^{n}+i \frac{\tau}{2} F^{n}\left(\psi^{n+1 / 2}\right), \quad n=0,1, \ldots,
$$

where $F^{n}: X_{M} \rightarrow X_{M}$ defined as

$$
\begin{aligned}
\left(F^{n}(u)\right)_{j k}= & {\left[-\frac{1}{2} \delta_{\nabla}^{2}+V_{j k}-\Omega L_{z}^{h}\right] u_{j k} } \\
& +\frac{\beta}{2}\left(\left|2 u_{j k}-\psi_{j, k}^{n}\right|^{2}+\left|\psi_{j, k}^{n}\right|^{2}\right) u_{j k}, \quad(j, k) \in \mathcal{T}_{M} .
\end{aligned}
$$

Define the map $G^{n}: X_{M} \rightarrow X_{M}$ as

$$
G^{n}(u)=u-\psi^{n}-i \frac{\tau}{2} F^{n}(u), \quad u \in X_{M},
$$

and it is easy to see that $G^{n}$ is continuous from $X_{M}$ to $X_{M}$. Moreover,

$$
\operatorname{Re}\left(G^{n}(u), u\right)=\|u\|_{2}^{2}-\operatorname{Re}\left(\psi^{n}, u\right) \geq\|u\|_{2}\left(\|u\|_{2}-\left\|\psi^{n}\right\|_{2}\right), \quad u \in X_{M},
$$


which immediately implies

$$
\lim _{\|u\|_{2} \rightarrow \infty} \frac{\left|\left(G^{n}(u), u\right)\right|}{\|u\|_{2}}=\infty .
$$

Thus $G^{n}$ is surjective. By using the Brouwer fixed point theorem (cf. [27]), it is easy to show that there exists a solution $u^{*}$ with $G^{n}\left(u^{*}\right)=0$, which implies that there exists a solution $\psi^{n+1 / 2}$ to the problem (4.2) and thus the CNFD discretization (2.1) is solvable for any given $\psi^{n}$. In addition, for the solution $\psi^{n+1}$ to (2.1), using (4.1), we have

$$
\left\|\delta_{\nabla}^{+} \psi^{n+1}\right\|_{2}^{2} \leq C E_{h}\left(\psi^{n+1}\right)=C E_{h}\left(\psi^{0}\right), \quad n=0,1, \ldots,
$$

where when $\beta \geq 0$, we have $C=2$, and when $\beta<0$ with $\left\|\psi^{0}\right\|_{2}^{2}<\frac{1}{|\beta|}\left(1-\frac{\Omega^{2}}{\gamma^{2}}\right)$, it comes from

$$
\begin{aligned}
E_{h}\left(\psi^{0}\right) & =E_{h}\left(\psi^{n+1}\right) \geq \frac{1}{2}\left(1-\frac{\Omega^{2}}{\gamma^{2}}\right)\left\|\delta_{\nabla}^{+} \psi^{n+1}\right\|_{2}^{2}-\frac{|\beta|}{2}\left\|\delta_{\nabla}^{+} \psi^{n+1}\right\|_{2}^{2} \cdot\left\|\psi^{n+1}\right\|_{2}^{2} \\
& =\frac{1}{2}\left(1-\frac{\Omega^{2}}{\gamma^{2}}\right)\left\|\delta_{\nabla}^{+} \psi^{n+1}\right\|_{2}^{2}-\frac{|\beta|}{2}\left\|\delta_{\nabla}^{+} \psi^{n+1}\right\|_{2}^{2} \cdot\left\|\psi^{0}\right\|_{2}^{2} \\
& =\frac{|\beta|}{2}\left[\frac{1}{|\beta|}\left(1-\frac{\Omega^{2}}{\gamma^{2}}\right)-\left\|\psi^{0}\right\|_{2}^{2}\right]\left\|\delta_{\nabla}^{+} \psi^{n+1}\right\|_{2}^{2} .
\end{aligned}
$$

Thus assume $h<1$, when $\beta \geq 0$ or $\beta<0$ with $\left\|\psi^{0}\right\|_{2}^{2}<\frac{1}{|\beta|}\left(1-\frac{\Omega^{2}}{\gamma^{2}}\right)$, using (4.3) and the inverse inequality [40, we obtain

$$
\left\|\psi^{n+1}\right\|_{\infty} \leq C|\ln h|\left\|\delta_{\nabla}^{+} \psi^{n+1}\right\|_{2} \leq C|\ln h| E_{h}\left(\psi^{0}\right), \quad n=0,1, \ldots .
$$

Next, we show the uniqueness of the solution of the CNFD scheme (2.1). For given $\psi^{n} \in X_{M}$, suppose that there are two solutions $u^{n+1} \in X_{M}$ and $v^{n+1} \in X_{M}$ to (2.1). From (4.4), we get

$$
\left\|u^{n+1}\right\|_{\infty} \leq C E_{h}\left(\psi^{0}\right)|\ln h|, \quad\left\|v^{n+1}\right\|_{\infty} \leq C E_{h}\left(\psi^{0}\right)|\ln h| .
$$

Denoting $w:=u^{n+1}-v^{n+1} \in X_{M}$, from (2.1), we have

$$
i \frac{w_{j k}}{\tau}=\left(-\frac{1}{2} \delta_{\nabla}^{2}+V_{j k}-\Omega L_{z}^{h}\right) w_{j k}+\hat{R}_{j k}, \quad(j, k) \in \mathcal{T}_{M},
$$

where

$\hat{R}_{j k}=\frac{\beta}{2}\left(\left|u_{i j}^{n+1}\right|^{2}+\left|\psi_{j k}^{n}\right|^{2}\right) w_{j k}+\frac{\beta}{2}\left(v_{j k}^{n+1}+\psi_{j k}^{n}\right)\left(\left|u_{j k}^{n+1}\right|^{2}-\left|v_{j k}^{n+1}\right|^{2}\right), \quad(j, k) \in \mathcal{T}_{M}$.

Multiplying both sides of (4.6) with $\bar{w}_{j k}$, summing for $(j, k) \in \mathcal{T}_{M}$, and then taking imaginary parts, using (4.4) and (4.5), we have

$$
\|w\|_{2}^{2} \leq \tau C\left[\left\|u^{n+1}\right\|_{\infty}^{2}+\left\|v^{n+1}\right\|_{\infty}^{2}+\left\|\psi^{n}\right\|_{\infty}^{2}\right]\|w\|_{2}^{2} \leq C \tau\left[E_{h}\left(\psi^{0}\right) \ln h\right]^{2}\|w\|_{2}^{2} .
$$

Thus under the assumption $\tau \lesssim h$, there exists $h_{0}>0$, when $0<h \leq h_{0}$, we have $C \tau\left(\ln h E_{h}\left(\psi^{0}\right)\right)^{2}<1$ which immediately implies

$$
\|w\|_{2}=\left\|u^{n+1}-v^{n+1}\right\|_{2}=0 \quad \Longrightarrow \quad u^{n+1}=v^{n+1},
$$

i.e., the solution of CNFD (2.1) is unique. 
Denote the local truncation error $\widetilde{\eta}^{n} \in X_{M}(n \geq 0)$ of the CNFD scheme (2.1) with (2.2) and (2.3) as

$$
\begin{array}{ll}
\widetilde{\eta}_{j k}^{n}:= & i \delta_{t}^{+} \psi\left(x_{j}, y_{k}, t_{n}\right)-\left[-\frac{1}{2} \delta_{\nabla}^{2}-\Omega L_{z}^{h}+V_{j k}+\frac{\beta}{2}\left(\left|\psi\left(x_{j}, y_{k}, t_{n+1}\right)\right|^{2}\right.\right. \\
& \left.\left.\quad+\left|\psi\left(x_{j}, y_{k}, t_{n}\right)\right|^{2}\right)\right] \times \frac{\psi\left(x_{j}, y_{k}, t_{n}\right)+\psi\left(x_{j}, y_{k}, t_{n+1}\right)}{2}, \quad(j, k) \in \mathcal{T}_{M} .
\end{array}
$$

Then we have

Lemma 4.3 (Local truncation error). Assume $V(\mathbf{x}) \in L^{\infty}(U)$ and under assumption (B), we have

$$
\left\|\widetilde{\eta}^{n}\right\|_{\infty} \lesssim \tau^{2}+h^{2}, \quad 0 \leq n \leq \frac{T}{\tau}-1 .
$$

In addition, assuming $V(\mathbf{x}) \in C^{1}(U)$ and $\tau \lesssim h$, we have for $1 \leq n \leq \frac{T}{\tau}-1$,

$$
\left|\delta_{\nabla}^{+} \widetilde{\eta}_{j k}^{n}\right| \lesssim \begin{cases}\tau^{2}+h^{2}, & 1 \leq j \leq M-2,1 \leq k \leq K-2, \\ \tau+h, & j=0, M-1, \text { or } k=0, K-1 .\end{cases}
$$

In addition, if either $\Omega=0$ and $V(\mathbf{x})=0$ or $\psi \in C^{0}\left([0, T] ; H_{0}^{2}(U)\right)$, we have

$$
\left\|\delta_{\nabla}^{+} \widetilde{\eta}^{n}\right\|_{\infty} \lesssim \tau^{2}+h^{2}, \quad 1 \leq n \leq \frac{T}{\tau}-1 .
$$

Proof. Follow the analogous line for Lemma 3.3 and we omit it here for brevity.

Theorem 4.1 ( $l^{2}$-norm estimate). Assume $\tau \lesssim h$ and either $\beta \geq 0$ or $\beta<0$ with $\left\|\psi^{0}\right\|_{2}^{2}<\frac{1}{|\beta|}\left(1-\frac{\Omega^{2}}{\gamma^{2}}\right)$, under assumptions $(\mathrm{A})$ and $(\mathrm{B})$, there exist $h_{0}>0$ and $\tau_{0}>0$ sufficiently small, when $0<h \leq h_{0}$ and $0<\tau \leq \tau_{0}$, we have

$$
\left\|e^{n}\right\|_{2} \lesssim \tau^{2}+h^{2}, \quad\left\|\psi^{n}\right\|_{\infty} \leq \sqrt{2}\left(1+M_{1}\right), \quad 0 \leq n \leq \frac{T}{\tau} .
$$

Proof. Choose a smooth function $\alpha(\rho)(\rho \geq 0) \in C^{\infty}([0, \infty))$ defined as

$$
\alpha(\rho)= \begin{cases}1, & 0 \leq \rho \leq 1, \\ \in[0,1], & 1 \leq \rho \leq 2, \\ 0, & \rho \geq 2\end{cases}
$$

Denote $M_{0}=2\left(1+M_{1}\right)^{2}>0$ and define

$$
F_{M_{0}}(\rho)=\alpha\left(\frac{\rho}{M_{0}}\right) \rho, \quad 0 \leq \rho<\infty,
$$

then $F_{M_{0}}(\rho) \in C^{\infty}([0, \infty))$ and it is global Lipschitz, i.e.,

$$
\left|F_{M_{0}}\left(\rho_{1}\right)-F_{M_{0}}\left(\rho_{2}\right)\right| \leq C_{M_{0}}\left|\sqrt{\rho_{1}}-\sqrt{\rho_{2}}\right|, \quad 0 \leq \rho_{1}, \rho_{2}<\infty .
$$

Choose $\phi^{0}=\psi^{0} \in X_{M}$ and define $\phi^{n} \in X_{M}(n=0,1, \ldots)$ as, for $(j, k) \in \mathcal{T}_{M}$,

$$
i \delta_{t}^{+} \phi_{j k}^{n}=\left[-\frac{1}{2} \delta_{\nabla}^{2}+V_{j k}-\Omega L_{z}^{h}+\frac{\beta}{2}\left(F_{M_{0}}\left(\left|\phi_{j k}^{n+1}\right|^{2}\right)+F_{M_{0}}\left(\left|\phi_{j k}^{n}\right|^{2}\right)\right)\right] \phi_{j k}^{n+1 / 2},
$$

where

$$
\phi_{j k}^{n+1 / 2}=\frac{1}{2}\left(\phi_{j k}^{n+1}+\phi_{j k}^{n}\right), \quad(j, k) \in \mathcal{T}_{M}^{0}, \quad n \geq 0
$$


In fact, $\phi^{n}$ can be viewed as another approximation of $\psi\left(\mathbf{x}, t_{n}\right)$. Define the "error" function $\hat{e}^{n} \in X_{M}$,

$$
\hat{e}_{j k}^{n}:=\psi\left(x_{j}, y_{k}, t_{n}\right)-\phi_{j k}^{n}, \quad(j, k) \in \mathcal{T}_{M}^{0}, \quad n \geq 0,
$$

and the local truncation error $\hat{\eta}^{n} \in X_{M}$ of the scheme (4.14) as

$$
\begin{aligned}
\hat{\eta}_{j k}^{n} & :=i \delta_{t}^{+} \psi\left(x_{j}, y_{k}, t_{n}\right)-\left[-\frac{1}{2} \delta_{\nabla}^{2}-\Omega L_{z}^{h}+V_{j k}+\frac{\beta}{2}\left(F_{M_{0}}\left(\left|\psi\left(x_{j}, y_{k}, t_{n+1}\right)\right|^{2}\right)\right.\right. \\
& \left.\left.+F_{M_{0}}\left(\left|\psi\left(x_{j}, y_{k}, t_{n}\right)\right|^{2}\right)\right)\right] \times \frac{\psi\left(x_{j}, y_{k}, t_{n}\right)+\psi\left(x_{j}, y_{k}, t_{n+1}\right)}{2}, \quad(j, k) \in \mathcal{T}_{M}, n \geq 0 .
\end{aligned}
$$

Similar to Lemma 4.3, we can prove

$$
\left\|\hat{\eta}^{n}\right\|_{\infty} \lesssim \tau^{2}+h^{2}, \quad 0 \leq n \leq \frac{T}{\tau} .
$$

Subtracting (4.15) from (4.14), we obtain

$$
\begin{aligned}
i \delta_{t}^{+} \hat{e}_{j, k}^{n} & =\left[-\frac{1}{2} \delta_{\nabla}^{2}+V_{j k}-\Omega L_{z}^{h}\right] \hat{e}_{j k}^{n+1 / 2} \\
& +\frac{\beta}{2}\left(F_{M_{0}}\left(\left|\phi_{j k}^{n+1}\right|^{2}\right)+F_{M_{0}}\left(\left|\phi_{j k}^{n}\right|^{2}\right)\right) \hat{e}_{j k}^{n+1 / 2} \\
& +\frac{\beta}{4}\left(\psi\left(x_{j}, y_{k}, t_{n+1}\right)+\psi\left(x_{j}, y_{k}, t_{n}\right)\right) \hat{\xi}_{j k}^{n}+\hat{\eta}_{j k}^{n}, \quad(j, k) \in \mathcal{T}_{M}, n \geq 0,
\end{aligned}
$$

where $\hat{\xi}^{n} \in X_{M}$ is defined as

$$
\begin{aligned}
\hat{\xi}_{j k}^{n}= & F_{M_{0}}\left(\left|\phi_{j k}^{n+1}\right|^{2}\right)+F_{M_{0}}\left(\left|\phi_{j k}^{n}\right|^{2}\right)-F_{M_{0}}\left(\left|\psi\left(x_{j}, y_{k}, t_{n+1}\right)\right|^{2}\right) \\
& -F_{M_{0}}\left(\left|\psi\left(x_{j}, y_{k}, t_{n}\right)\right|^{2}\right),(j, k) \in \mathcal{T}_{M}^{0} .
\end{aligned}
$$

This together with (4.13) implies

$$
\left|\frac{\beta}{4}\left(\psi\left(x_{j}, y_{k}, t_{n+1}\right)+\psi\left(x_{j}, y_{k}, t_{n}\right)\right) \hat{\xi}_{j k}^{n}\right| \lesssim C\left(\left|\hat{e}_{j k}^{n+1}\right|+\left|\hat{e}_{j k}^{n}\right|\right), \quad(j, k) \in \mathcal{T}_{M}^{0} .
$$

Multiplying both sides of (4.16) with $\overline{\hat{e}_{j k}^{n+1}+\hat{e}_{j k}^{n}}$, summing for $(j, k) \in \mathcal{T}_{M}$, taking imaginary part and applying the Cauchy inequality, we obtain

$$
\begin{aligned}
\left\|\hat{e}^{n+1}\right\|_{2}^{2}-\left\|\hat{e}^{n}\right\|_{2}^{2} & \lesssim \tau\left(\left|\hat{\eta}^{n}\right|_{\infty}^{2}+C\left(\left\|\hat{e}^{n+1}\right\|_{2}^{2}+\left\|\hat{e}^{n}\right\|_{2}^{2}\right)\right) \\
& \lesssim \tau\left[\left(h^{2}+\tau^{2}\right)^{2}+\left(\left\|\hat{e}^{n+1}\right\|_{2}^{2}+\left\|\hat{e}^{n}\right\|_{2}^{2}\right)\right], \quad 0 \leq n \leq \frac{T}{\tau}-1 .
\end{aligned}
$$

Then there exists $\tau_{0}>0$ sufficiently small, when $0<\tau \leq \tau_{0}$, applying the discrete Gronwall inequality [16, 21, 28, we get

$$
\left\|\hat{e}^{n}\right\|_{2} \lesssim \tau^{2}+h^{2}, \quad 0 \leq n \leq \frac{T}{\tau} .
$$

Applying the inverse inequality in $2 \mathrm{D}$, we have

$$
\left\|\hat{e}^{n}\right\|_{\infty} \lesssim \frac{1}{h}\left\|\hat{e}^{n}\right\|_{2} \lesssim h+\frac{\tau^{2}}{h} \lesssim h, \quad 0 \leq n \leq \frac{T}{\tau}
$$

which implies

$$
\left\|\phi^{n}\right\|_{\infty} \leq\left\|\Pi_{h} \psi\left(t_{n}\right)\right\|_{\infty}+\left\|\hat{e}^{n}\right\|_{\infty} \leq \frac{\sqrt{M_{0}}}{2}+C h, \quad 0 \leq n \leq \frac{T}{\tau} .
$$


Thus under the assumption $\tau \lesssim h$, there exists $h_{0}>0$, when $0<h \leq h_{0}$, we have (4.18)

$$
\left\|\phi^{n}\right\|_{\infty} \leq \frac{\sqrt{M_{0}}}{2}+\frac{\sqrt{M_{0}}}{2}=\sqrt{M_{0}} \quad \Longrightarrow \quad\left\|\phi^{n}\right\|_{\infty}^{2} \leq M_{0}, \quad 0 \leq n \leq \frac{T}{\tau} .
$$

Therefore, the discretization (4.14) collapses exactly to the CNFD discretization (2.1) with (2.2) and (2.3), i.e.,

$$
\psi^{n}=\phi^{n}, \quad e^{n}=\hat{e}^{n}, \quad 0 \leq n \leq \frac{T}{\tau} .
$$

This together with (4.17) and (4.18) complete the proof.

Again, combining Theorem 4.1 and Lemmas 4.2 and 4.3 , we are now ready to prove the main Theorem 2.2

Proof of Theorem 2.2. As in the proof of Theorem 2.1 we only prove the optimal convergence under assumptions (A) and (B) with either $\Omega=0$ and $V(\mathbf{x})=0$ or $\psi \in C^{0}\left([0, T] ; H_{0}^{2}(U)\right)$. Subtracting (4.7) from (2.1), we get

$$
i \delta_{t}^{+} e_{j k}^{n}=\left[-\frac{1}{2} \delta_{\nabla}^{2}+V_{j k}-\Omega L_{z}^{h}\right] e_{j k}^{n+1 / 2}+\widetilde{\xi}_{j k}^{n}+\widetilde{\eta}_{j k}^{n}, \quad(j, k) \in \mathcal{T}_{M}, n \geq 0,
$$

where $\tilde{\xi}^{n} \in X_{M}$ is defined as

$$
\begin{aligned}
\widetilde{\xi}_{j k}^{n}= & \frac{\beta}{2}\left[e_{j k}^{n} \overline{\psi\left(x_{j}, y_{k}, t_{n}\right)}+\psi_{j k}^{n} \overline{e_{j k}^{n}}+e_{j k}^{n+1} \overline{\psi\left(x_{j}, y_{k}, t_{n+1}\right)}+\psi_{j k}^{n+1} \overline{e_{j k}^{n+1}}\right] \psi_{j k}^{n+1 / 2} \\
& +\frac{\beta}{2}\left(\left|\psi\left(x_{j}, y_{k}, t_{n}\right)\right|^{2}+\left|\psi\left(x_{j}, y_{k}, t_{n+1}\right)\right|^{2}\right) e_{j k}^{n+1 / 2}, \quad(j, k) \in \mathcal{T}_{M} .
\end{aligned}
$$

Again, rewrite (4.19) as

$$
e^{n+1}-e^{n}=-i \tau\left(\widetilde{\chi}^{n}+\widetilde{\xi}^{n}+\widetilde{\eta}^{n}\right), \quad n \geq 0,
$$

where $\tilde{\chi}^{n} \in X_{M}$ is defined as

$$
\tilde{\chi}_{j k}^{n}=\left[-\frac{1}{2} \delta_{\nabla}^{2}+V_{j k}-\Omega L_{z}^{h}\right] e_{j k}^{n+1 / 2}, \quad(j, k) \in \mathcal{T}_{M}, \quad n \geq 0 .
$$

Multiplying both sides of (4.19) with $\overline{e_{j k}^{n+1}-e_{j k}^{n}}$, summing for $(j, k) \in \mathcal{T}_{M}$, noticing (3.1), (3.2) and (4.20), taking real parts, we obtain

$$
\begin{aligned}
\mathcal{E}\left(e^{n+1}\right)-\mathcal{E}\left(e^{n}\right) & =-2 \operatorname{Re}\left\langle\widetilde{\xi}^{n}+\widetilde{\eta}^{n}, e^{n+1}-e^{n}\right\rangle \\
& =-2 \operatorname{Re}\left\langle\widetilde{\xi}^{n}+\widetilde{\eta}^{n},-i \tau\left(\widetilde{\chi}^{n}+\widetilde{\xi}^{n}+\widetilde{\eta}^{n}\right)\right\rangle \\
& =2 \tau \operatorname{Im}\left\langle\widetilde{\xi}^{n}+\widetilde{\eta}^{n}, \widetilde{\chi}^{n}\right\rangle, \quad 0 \leq n \leq \frac{T}{\tau}-1 .
\end{aligned}
$$


Similar to those in the proof of Theorem 2.1, we can prove

$$
\left|\operatorname{Im}\left\langle\widetilde{\xi}^{n}+\widetilde{\eta}^{n}, \widetilde{\chi}^{n}\right\rangle\right| \lesssim\left(h^{2}+\tau^{2}\right)^{2}+\mathcal{E}\left(e^{n+1}\right)+\mathcal{E}\left(e^{n}\right), \quad 0 \leq n \leq \frac{T}{\tau}-1 .
$$

Combining the above two inequalities, we get

$$
\mathcal{E}\left(e^{n+1}\right)-\mathcal{E}\left(e^{n}\right) \lesssim \tau\left[\left(\tau^{2}+h^{2}\right)^{2}+\mathcal{E}\left(e^{n+1}\right)+\mathcal{E}\left(e^{n}\right)\right], \quad 0 \leq n \leq \frac{T}{\tau}-1
$$

Then there exists $\tau_{0}>0$ sufficiently small, when $0<\tau \leq \tau_{0}$, using the discrete Gronwall inequality [16, 21, 28, and noticing $e^{0}=0$ and $\mathcal{E}\left(e^{0}\right)=0$, we get

$$
\mathcal{E}\left(e^{n}\right) \lesssim\left(\tau^{2}+h^{2}\right)^{2}, \quad 0 \leq n \leq \frac{T}{\tau},
$$

which immediately implies (2.14). If we only have assumptions (A) and (B) without further assumption, the convergence rate in the semi- $H^{1}$ norm will be $O\left(h^{3 / 2}+\right.$ $\left.\tau^{3 / 2}\right)$. The proof is the same as in Theorem 2.1, and we omit it here.

Similarly, from Theorem 2.2 and using the inverse inequality [40, we get immediately the error estimate in $l^{\infty}$-norm for the CNFD method as

Lemma 4.4 ( $l^{\infty}$-norm estimate). Under the same conditions of Theorem 2.2 with assumptions (A) and (B) and assume $h<1$, we have the following error estimate for the CNFD:

$$
\left\|e^{n}\right\|_{\infty} \lesssim \begin{cases}\left(h^{3 / 2}+\tau^{3 / 2}\right)|\ln (h)|, & d=2, \\ h+\tau, & d=3 .\end{cases}
$$

In addition, if either $\Omega=0$ and $V(\mathbf{x})=0$ or $\psi \in C^{0}\left([0, T] ; H_{0}^{2}(U)\right)$, we have

$$
\left\|e^{n}\right\|_{\infty} \lesssim \begin{cases}\left(h^{2}+\tau^{2}\right)|\ln (h)|, & d=2, \\ h^{3 / 2}+\tau^{3 / 2}, & d=3 .\end{cases}
$$

Remark 4.1. If the cubic nonlinear term $\beta|\psi|^{2} \psi$ in (1.1) is replaced by a general nonlinearity $f\left(|\psi|^{2}\right) \psi$, the numerical discretization CNFD and its error estimates in $l^{2}$-norm, $l^{\infty}$-norm and discrete $H^{1}$-norm are still valid provided that the nonlinear real-valued function $f(\rho) \in C^{3}([0, \infty))$.

\section{EXTENSION TO OTHER CASES}

In this section, we will discuss a discretization of the GPE with an angular momentum rotation (1.1) when $U$ is a disk in 2D, and resp., a cylinder in 3D and its error estimates. As noticed in [6], the angular momentum rotation is constant coefficient in $2 \mathrm{D}$ with polar coordinates and $3 \mathrm{D}$ with cylindrical coordinates. Thus the original problem of GPE with an angular momentum rotation term defined in $\mathbb{R}^{d}(d=2,3)$ for rotating BEC is usually truncated onto a disk in $2 \mathrm{D}$ and a cylinder in $3 \mathrm{D}$ as bounded computational domain. Again, for simplicity of notation, we only consider SIFD in 2D, i.e., $d=2$ and $U=\{\mathbf{x}|| \mathbf{x} \mid<R\}$ with $R>0$ fixed. Extension to $3 \mathrm{D}$ are straightforward. In $2 \mathrm{D}$ with polar coordinate, the problem collapses

$$
i \partial_{t} \psi=\left[-\frac{1}{2}\left(\frac{1}{r} \partial_{r}\left(r \partial_{r}\right)+\frac{1}{r^{2}} \partial_{\theta \theta}\right)+V_{0}(r)+W(r, \theta)+i \Omega \partial_{\theta}+\beta|\psi|^{2}\right] \psi, \quad(r, \theta) \in U,
$$


with boundary condition

$$
\psi(R, \theta)=0, \quad \psi(r, \theta)=\psi(r, \theta+2 \pi), \quad 0 \leq \theta \leq 2 \pi, \quad 0 \leq r \leq R,
$$

and initial condition

$$
\psi(r, \theta, 0)=\psi_{0}(r, \theta), \quad 0 \leq r \leq R, \quad 0 \leq \theta \leq 2 \pi,
$$

where $\psi=\psi(r, \theta, t)$ and here we split the external trapping potential $V(\mathbf{x})$ into a radial symmetry part $V_{0}(r)$ and a left-over part $W(\mathbf{x})$, i.e.,

$$
V(\mathbf{x})=V_{0}(r)+W(r, \theta), \quad \mathbf{x} \in U .
$$

Let $M, K>0$ be two positive integers, and $\Delta r:=\frac{2 R}{2 M+1}, \Delta \theta:=\frac{2 \pi}{K}$, define the grid points

$$
\begin{gathered}
r_{j}=j \Delta r, \quad r_{j+\frac{1}{2}}=\left(j+\frac{1}{2}\right) \Delta r, \quad j=0,1, \ldots, M ; \\
\theta_{k}=k \Delta \theta, \quad k=0,1, \ldots, K .
\end{gathered}
$$

Let $\psi_{j+\frac{1}{2} k}^{n}$ be the approximation of $\psi\left(r_{j+\frac{1}{2}}, \theta_{k}, t_{n}\right)$ and $\psi^{n}$ be the numerical solution at time $t=t_{n}$. We adopt similar notations as those in section 2 .

Then a semi-implicit finite difference (SIFD) discretization reads for $n \geq 1$

$$
\begin{aligned}
& i \delta_{t} \psi_{j+\frac{1}{2} k}^{n}=\frac{-r_{j+\frac{1}{2}}^{-1}}{4(\Delta r)^{2}}\left[r_{j+1}\left(\psi_{j+\frac{3}{2} k}^{n+1}+\psi_{j+\frac{3}{2} k}^{n-1}\right)\right. \\
& \quad-\left(r_{j+1}+r_{j}\right)\left(\psi_{j+\frac{1}{2} k}^{n+1}+r_{j}\left(\psi_{j-\frac{1}{2} k}^{n+1}+\psi_{j-\frac{1}{2} k}^{n-1}\right)\right] \\
& \quad-\frac{1}{4 r_{j+\frac{1}{2}}^{2}(\Delta \theta)^{2}}\left[\psi_{j+\frac{1}{2} k+1}^{n+1}-2 \psi_{j+\frac{1}{2} k}^{n+1}+\psi_{j+\frac{1}{2} k-1}^{n+1}+\psi_{j+\frac{1}{2} k+1}^{n-1}-2 \psi_{j+\frac{1}{2} k}^{n-1}+\psi_{j+\frac{1}{2} k-1}^{n-1}\right] \\
& \quad+\frac{V_{0}\left(r_{j+\frac{1}{2}}\right)}{2}\left(\psi_{j+\frac{1}{2} k}^{n+1}+\psi_{j+\frac{1}{2} k}^{n-1}\right)+\frac{i \Omega}{2 \Delta \theta}\left[\psi_{j+\frac{1}{2} k+1}^{n+1}-\psi_{j+\frac{1}{2} k-1}^{n+1}+\psi_{j+\frac{1}{2} k+1}^{n-1}-\psi_{j+\frac{1}{2} k-1}^{n-1}\right] \\
& +\beta\left|\psi_{j+\frac{1}{2} k}^{n}\right|^{2} \psi_{j+\frac{1}{2} k}^{n}+W\left(r_{j+\frac{1}{2}}, \theta_{k}\right) \psi_{j+\frac{1}{2} k}^{n}, \quad 0 \leq j \leq M-1,0<k \leq K .
\end{aligned}
$$

The boundary condition (5.2) is discretized as

$$
\psi_{M+\frac{1}{2} k}=0,0 \leq k \leq K, \quad \psi_{j+\frac{1}{2} 0}=\psi_{j+\frac{1}{2} K}, \psi_{j+\frac{1}{2} K+1}=\psi_{j+\frac{1}{2} 1}, \quad 0 \leq j \leq M,
$$

and the initial condition (5.3) is discretized as

$$
\psi_{j+\frac{1}{2} k}^{0}=\psi_{0}\left(r_{j+\frac{1}{2}}, \theta_{k}\right), \quad 0 \leq j \leq M, \quad 0 \leq k \leq K .
$$

The first step $\psi^{1}$ can be obtained by using the same spatial discretization combining with any explicit second-order time integrator.

For this SIFD method, although it is implicit, however, at each time step, the linear system can be solved directly via fast direct Poisson solver via fast discrete Fourier transform in $\theta$-direction with computational cost at $O(M K \ln K)$, i.e., it is very efficient in practical computation [6. In fact, this method is also widely used in simulating quantized vortex dynamics of rotating Bose-Einstein condensate [6]. In addition, let $e_{j+1 / 2 k}^{n}=\psi_{j+1 / 2 k}^{n}-\psi\left(r_{j+\frac{1}{2}}, \theta_{k}, t_{n}\right)$, similar to those in section 3 , we can prove the following error estimate. 
Theorem 5.1. Assume $h:=h_{\max }=\max \{\Delta r, \Delta \theta\} \lesssim h_{\min }:=\min \{\Delta r, \Delta \theta\}$ and $\tau \lesssim h$, under assumptions (A) and (B), there exist $h_{0}>0$ and $0<\tau_{0}<\frac{1}{4}$ sufficiently small, when $0<h \leq h_{0}$ and $0<\tau \leq \tau_{0}$, we have the following optimal error estimate for the SIFD method (5.4) with (5.5), (5.6)

$$
\left\|e^{n}\right\|_{2} \lesssim h^{2}+\tau^{2}, \quad\left\|\delta_{\nabla}^{+} e^{n}\right\|_{2} \lesssim h^{3 / 2}+\tau^{3 / 2}, \quad 0 \leq n \leq \frac{T}{\tau},
$$

where

$$
\begin{aligned}
& \left\|e^{n}\right\|_{2}^{2}=\Delta r \Delta \theta \sum_{j=0}^{M-1} \sum_{k=0}^{K-1} r_{j+\frac{1}{2}}\left|e_{j+\frac{1}{2} k}^{n}\right|^{2}, \quad n=0,1, \ldots, \\
& \left\|\delta_{\nabla}^{+} e^{n}\right\|_{2}^{2}=\Delta r \Delta \theta \sum_{j=0}^{M-1} \sum_{k=0}^{K-1}\left[r_{j+1}\left|\frac{e_{j+\frac{3}{2} k}^{n}-e_{j+\frac{1}{2} k}^{n}}{\Delta r}\right|^{2}\right. \\
& \left.+\frac{1}{r_{j+\frac{1}{2}}}\left|\frac{e_{j+\frac{1}{2} k+1}^{n}-e_{j+\frac{1}{2} k}^{n}}{\Delta \theta}\right|^{2}\right] \text {. }
\end{aligned}
$$

In addition, assuming $\psi \in C^{0}\left([0, T] ; H_{0}^{2}(U)\right)$, we have

$$
\left\|e^{n}\right\|_{2}+\left\|\delta_{\nabla}^{+} e^{n}\right\|_{2} \lesssim h^{2}+\tau^{2}, \quad 0 \leq n \leq \frac{T}{\tau} .
$$

The CNFD method and its error estimate can be extended to this case directly and we omit the details for brevity. Again, it is implicit and at every time step, a nonlinear system must be solved.

\section{NUMERICAL RESUlTS}

In this section, we report numerical results of the SIFD (2.4) and CNFD (2.1) discretizations of the GPE (1.1) to confirm the error estimates.

We take $d=2, U=[-8,8] \times[-8,8], V(\mathbf{x})=\frac{1}{2}\left(x^{2}+y^{2}\right), \beta=10$ in (1.1) and $\psi_{0}(\mathbf{x})=\frac{2}{\sqrt{\pi}}(x+i y) e^{-\left(x^{2}+y^{2}\right)}$ in (1.3). For comparison, the numerical "exact" solution $\psi_{e}$ is obtained by the CNFD with a very fine mesh and a small time step, e.g., $h=1 / 64$ and $\tau=0.0001$. For SIFD scheme, at each time step, we use GaussSeidel iteration method to solve the linear system. For CNFD scheme, to solve the fully nonlinear system, at each iteration, the system is linearized, i.e., the CNFD (2.1) is linearized as

$$
\begin{aligned}
& i \frac{\psi_{j k}^{(m)}-\psi_{j k}^{n}}{\tau} \\
& \quad=\left[-\frac{1}{2} \delta_{\nabla}^{2}+V_{j k}-\Omega L_{z}^{h}+\frac{\beta}{2}\left(\left|\psi_{j k}^{n}\right|^{2}+\left|\psi_{j k}^{(m-1)}\right|^{2}\right)\right] \frac{1}{2}\left(\psi_{j k}^{(m)}+\psi_{j k}^{n}\right), m \geq 1,
\end{aligned}
$$

and we solve this inner problem to get $\psi_{j k}^{(m)}$ by Gauss-Seidel iteration method. Then the solution $\psi_{j k}^{n+1}$ is numerically reached once $\psi_{j k}^{(m)}$ converges. 
Let $\psi_{h, \tau}$ be the numerical solution corresponding to mesh size $h$ and time step $\tau$ and define the error function as $e:=\psi_{e}-\psi_{h, \tau}$. The convergence rates are calculated as $\log _{2}(\|e(h, \tau)\| /\|e(h / 2, \tau / 2)\|)$ with the corresponding norms. Table 1 shows the errors $\|e\|_{2},\left\|\delta_{\nabla}^{+} e\right\|_{2}$ and $\|e\|_{\infty}$ for the CNFD method (2.1) with different $\Omega, h$ and $\tau$, and Table 2 displays similar results for SIFD method (2.4). Figures 1 and 2 depict time evolution of the errors between the discretized mass and energy with their continuous counterparts, respectively, i.e., $\left|\left\|\psi^{n}\right\|_{2}^{2}-N\left(\psi_{0}\right)\right|$ and $\left|E_{h}\left(\psi^{n}\right)-E\left(\psi_{0}\right)\right|$ of the SIFD method (2.4) for different $\Omega, h$ and $\tau$. Figure 3 displays similar results of the CNFD method (2.1) when the nonlinear system is iteratively solved up to a given accuracy $\varepsilon>0$.

From Tables 1 and 2, we demonstrate the second-order convergence rate of both SIFD and CNFD methods in $l^{2}$-norm, $l^{\infty}$-norm and discrete $H^{1}$-norm. From Figures 1,2 and 3, we can draw the following conclusions:

(i) the SIFD discretization approximates the mass very well (up to 4 significant digits, cf. Figure 1) and the energy at second order accurate in practical computation when $\tau=O(h)$ are not too big (cf. Figure 1). When the final computational time $t$ increases, the errors in mass or energy are either oscilatting or slightly increasing (cf. Figures 1 and 2). An interesting observation is that, for fixed $h>0$ small, when $\tau>0$ very small, the errors in mass and energy increase with time, especially in long time (cf. Figure 2).

(ii) For the CNFD discretization, when the fully nonlinear system is iteratively solved at every time step to extremely high accuracy, e.g., machine accuracy, the solution obtained in practical computation conserves the mass and energy very well (cf. Figure 3). However, if the nonlinear system is solved accurately but not extremely accurately, the solution obtained in practical computation does not conserve the mass and energy very well, especially in long time (cf. Figure 3).

(iii) From the accuracy point of view, SIFD method is the same accurate as CNFD method and it approximates the mass very well and the energy in the same order as the numerical solution in the discretized level. It is much cheaper than CNFD method, especially in high dimensions and/or when fast Poisson solver is applied in practical computation. 
TABLE 1. Error analysis of the CNFD method (2.1) for the GPE (1.1) at time $t=0.5$ for different $\Omega$, mesh size $h$ and time step $\tau$.

\begin{tabular}{|c|c|c|c|c|c|}
\hline \multirow{5}{*}{$\Omega=0$} & & $h=1 / 4$ & $h=1 / 8$ & $h=1 / 16$ & $h=1 / 32$ \\
& & $\tau=2^{-5}$ & $\tau=2^{-6}$ & $\tau=2^{-7}$ & $\tau=2^{-8}$ \\
\hline \multirow{5}{*}{$\Omega=0.5$} & $\|e\|_{2}$ & $5.424 \mathrm{E}-2$ & $1.574 \mathrm{E}-2$ & $3.907 \mathrm{E}-3$ & $8.268 \mathrm{E}-4$ \\
& Rate & 1.78 & 2.01 & 2.24 & \\
\cline { 2 - 6 } & $\left\|\delta_{\nabla}^{+} e\right\|_{2}$ & $2.257 \mathrm{E}-1$ & $8.008 \mathrm{E}-2$ & $2.066 \mathrm{E}-2$ & $4.448 \mathrm{E}-3$ \\
& Rate & 1.50 & 1.95 & 2.22 & \\
\cline { 2 - 6 } & $\|e\|_{\infty}$ & $1.521 \mathrm{E}-2$ & $3.273 \mathrm{E}-3$ & $7.676 \mathrm{E}-3$ & $1.585 \mathrm{E}-4$ \\
& Rate & 2.22 & 2.09 & 2.28 & \\
\hline \multirow{5}{*}{$\Omega=0.9$} & $\|e\|_{2}$ & $4.758 \mathrm{E}-2$ & $1.408 \mathrm{E}-2$ & $3.502 \mathrm{E}-3$ & $7.425 \mathrm{E}-4$ \\
& Rate & 1.76 & 2.01 & 2.24 & \\
\cline { 2 - 6 } & $\left\|\delta_{\nabla}^{+} e\right\|_{2}$ & $2.097 \mathrm{E}-1$ & $7.535 \mathrm{E}-2$ & $1.943 \mathrm{E}-2$ & $4.186 \mathrm{E}-3$ \\
& Rate & 1.48 & 1.96 & 2.21 & \\
\cline { 2 - 6 } & $\|e\|_{\infty}$ & $1.259 \mathrm{E}-2$ & $3.081 \mathrm{E}-3$ & $7.233 \mathrm{E}-4$ & $1.489 \mathrm{E}-4$ \\
& Rate & 2.03 & 2.09 & 2.28 & \\
\hline & $\|e\|_{2}$ & $4.406 \mathrm{E}-2$ & $1.315 \mathrm{E}-2$ & $3.272 \mathrm{E}-3$ & $6.934 \mathrm{E}-4$ \\
& Rate & 1.74 & 2.01 & 2.24 & \\
\cline { 2 - 6 } & $\left\|\delta_{\nabla}^{+} e\right\|_{2}$ & $2.007 \mathrm{E}-1$ & $7.240 \mathrm{E}-2$ & $1.863 \mathrm{E}-2$ & $4.011 \mathrm{E}-3$ \\
& Rate & 1.47 & 1.96 & 2.22 & \\
\cline { 2 - 6 } & $\|e\|_{\infty}$ & $1.196 \mathrm{E}-2$ & $3.105 \mathrm{E}-3$ & $7.284 \mathrm{E}-4$ & $1.494 \mathrm{E}-4$ \\
& Rate & 1.95 & 2.09 & 2.29 & \\
\hline
\end{tabular}

TABLE 2. Error analysis of the SIFD method (2.4) for the GPE (1.1) at time $t=0.5$ for different $\Omega$, mesh size $h$ and time step $\tau$.

\begin{tabular}{|c|c|c|c|c|c|}
\hline \multirow{5}{*}{$\Omega=0$} & & $h=1 / 4$ & $h=1 / 8$ & $h=1 / 16$ & $h=1 / 32$ \\
& & $\tau=2^{-7}$ & $\tau=2^{-8}$ & $\tau=2^{-9}$ & $\tau=2^{-10}$ \\
\hline \multirow{5}{*}{$\Omega=0.5$} & $\|e\|_{2}$ & $4.943 \mathrm{E}-2$ & $1.360 \mathrm{E}-2$ & $3.285 \mathrm{E}-3$ & $6.661 \mathrm{E}-4$ \\
& Rate & 1.92 & 1.99 & 2.30 & \\
\cline { 2 - 6 } & $\left\|\delta_{\nabla}^{+} e\right\|_{2}$ & $2.084 \mathrm{E}-1$ & $6.726 \mathrm{E}-2$ & $1.663 \mathrm{E}-2$ & $3.399 \mathrm{E}-3$ \\
& Rate & 1.63 & 2.02 & 2.29 & \\
\cline { 2 - 6 } & $\|e\|_{\infty}$ & $1.298 \mathrm{E}-2$ & $2.867 \mathrm{E}-3$ & $6.709 \mathrm{E}-4$ & $1.346 \mathrm{E}-4$ \\
& Rate & 2.18 & 2.10 & 2.32 & \\
\hline \multirow{5}{*}{$\Omega=0.9$} & $\|e\|_{2}$ & $4.350 \mathrm{E}-2$ & $1.212 \mathrm{E}-2$ & $2.927 \mathrm{E}-3$ & $5.938 \mathrm{E}-4$ \\
& Rate & 1.84 & 2.05 & 2.30 & \\
\cline { 2 - 6 } & $\left\|\delta_{\nabla}^{+} e\right\|_{2}$ & $1.940 \mathrm{E}-1$ & $6.319 \mathrm{E}-2$ & $1.561 \mathrm{E}-2$ & $3.191 \mathrm{E}-3$ \\
& Rate & 1.62 & 2.02 & 2.29 & \\
\cline { 2 - 6 } & $\|e\|_{\infty}$ & $1.165 \mathrm{E}-2$ & $2.748 \mathrm{E}-3$ & $6.449 \mathrm{E}-4$ & $1.295 \mathrm{E}-4$ \\
& Rate & 2.08 & 2.09 & 2.32 & \\
\hline & $\|e\|_{2}$ & $4.060 \mathrm{E}-2$ & $1.136 \mathrm{E}-2$ & $2.741 \mathrm{E}-3$ & $5.557 \mathrm{E}-4$ \\
& Rate & 1.84 & 2.05 & 2.30 & \\
\cline { 2 - 6 } & $\left\|\delta_{\nabla}^{+} e\right\|_{2}$ & $1.863 \mathrm{E}-1$ & $6.085 \mathrm{E}-2$ & $1.499 \mathrm{E}-2$ & $3.062 \mathrm{E}-3$ \\
& Rate & 1.61 & 2.02 & 2.29 & \\
\cline { 2 - 6 } & $\|e\|_{\infty}$ & $1.101 \mathrm{E}-2$ & $2.726 \mathrm{E}-3$ & $6.339 \mathrm{E}-4$ & $1.271 \mathrm{E}-4$ \\
& Rate & 2.01 & 2.10 & 2.32 & \\
\hline
\end{tabular}



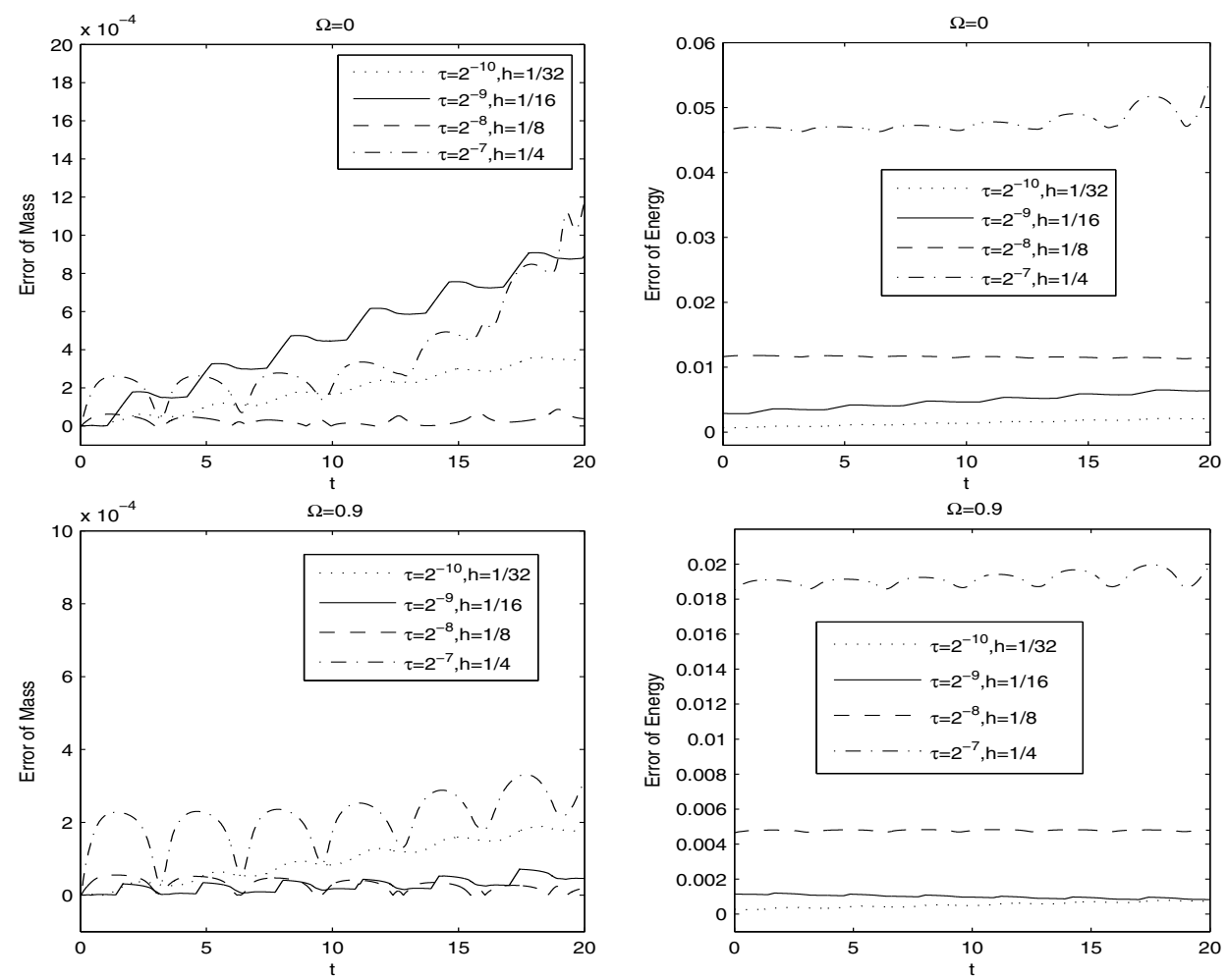

Figure 1. Time evolution of the errors between the discretized mass and energy with their continuous counterparts, i.e., $\left|\left\|\psi^{n}\right\|_{2}^{2}-N\left(\psi_{0}\right)\right|$ and $\left|E_{h}\left(\psi^{n}\right)-E\left(\psi_{0}\right)\right|$, of the SIFD scheme (2.4) for different $\Omega$ and $\tau=O(h)$. 

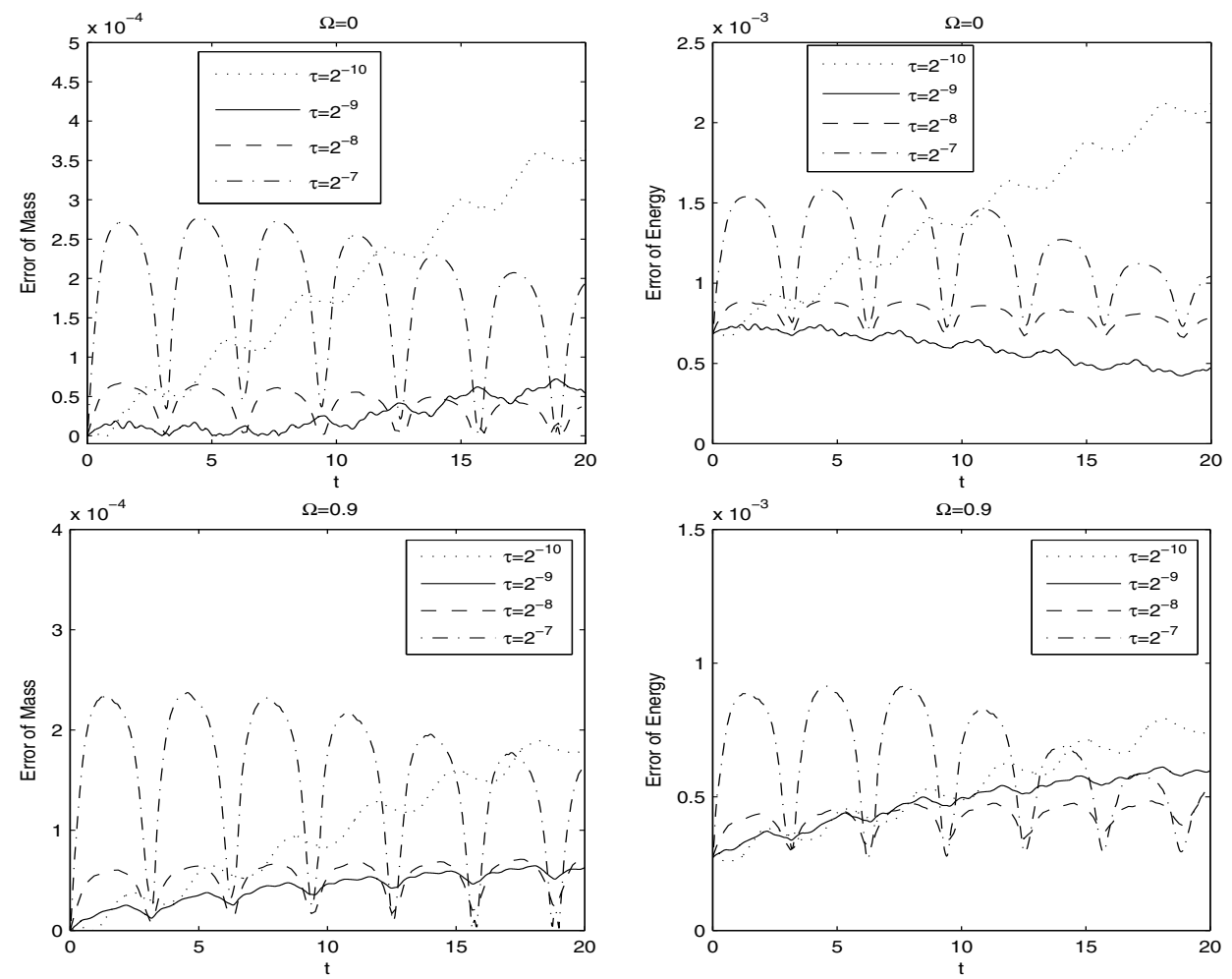

Figure 2. Time evolution of the errors between the discretized mass and energy with their continuous counterparts, i.e., $\left|\left\|\psi^{n}\right\|_{2}^{2}-N\left(\psi_{0}\right)\right|$ and $\left|E_{h}\left(\psi^{n}\right)-E\left(\psi_{0}\right)\right|$, of the SIFD scheme (2.4) with $h=1 / 32$ for different $\Omega$ and time steps $\tau$. 

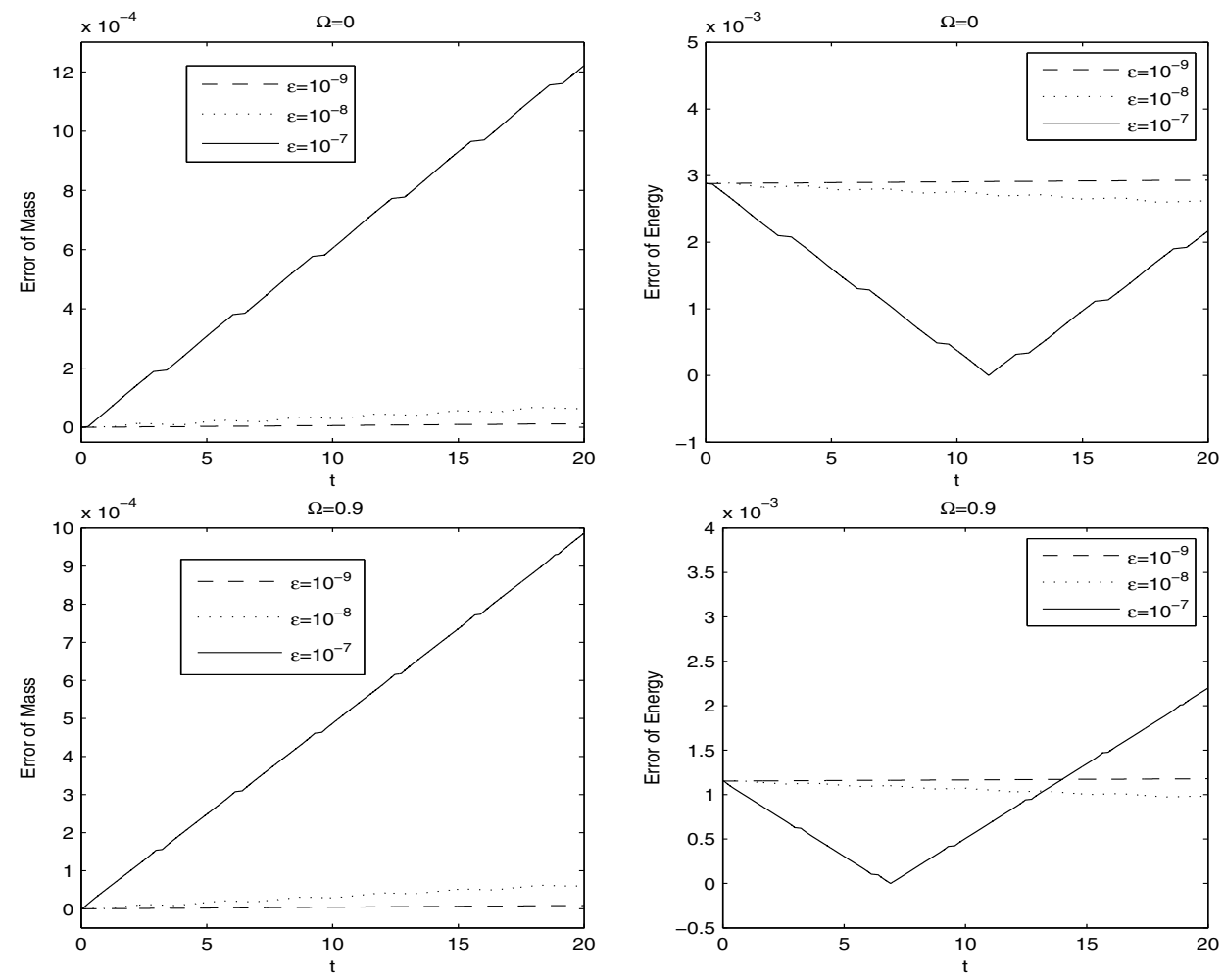

Figure 3. Time evolution of the errors between the discretized mass and energy with their continuous counterparts, i.e., $\left|\left\|\psi^{n}\right\|_{2}^{2}-N\left(\psi_{0}\right)\right|$ and $\left|E_{h}\left(\psi^{n}\right)-E\left(\psi_{0}\right)\right|$, of the CNFD scheme (2.1) with mesh $h=1 / 16$ and time step $\tau=2^{-9}$ when the nonlinear system is iteratively solved up to the accuracy $\varepsilon$ for different $\Omega$ and $\varepsilon$.

\section{Conclusions}

We carried out rigorous numerical analysis on the conservative Crank-Nicolson finite difference (CNFD) method and semi-implicit finite difference (SIFD) method for discretizing the Gross-Pitaevskii equation (GPE) with an angular momentum rotation in two and three dimensions for rotating Bose-Einstein condensates (BEC). For both CNFD and SIFD, we obtained optimal convergence rate at the order of $O\left(h^{2}+\tau^{2}\right)$ in $l^{2}$-norm and discrete $H^{1}$-norm with time step $\tau$ and mesh size $h$. In the proof for the SIFD method, we made use of the method of mathematical induction, and for the CNFD scheme, we obtained an a priori bound of the numerical solution in the $l^{\infty}$-norm by approximating the nonlinearity with a Lipschitz function and using the inverse inequality. Numerical results confirmed our error estimates. In practice, the CNFD is unconditionally stable and it conserves mass and energy in the discretized level, however, it is implicit and a fully nonlinear system needs to be solved at every time step, which may be very expensive in $2 \mathrm{D}$ and/or $3 \mathrm{D}$. The SIFD is conditionally stable and it conserves the mass and energy well when $h=O(\tau)$ small, and a linear system needs to be solved at every time step. In 
addition, when the computational domain is a disk in $2 \mathrm{D}$, and resp., a cylinder in $3 \mathrm{D}$, the SIFD discretization can be extremely efficient in practical computation by using polar coordinates in $2 \mathrm{D}$, and resp., cylindrical coordinates in $3 \mathrm{D}$, together with fast direct Poisson solver. A similar idea to this method has been used in simulating quantized vortex dynamics in rotating BEC [6, 9, 12.

\section{ACKNOWLEDGEMENTS}

This work was supported by the Academic Research Fund of Ministry of Education of Singapore grant R-146-000-120-112. We acknowledge very stimulating and helpful discussions with Professor John W. Barrett on the topic. This work was partially done while the authors were visiting the Institute for Mathematical Sciences, National University of Singapore, in 2009.

\section{REFERENCES}

[1] J. R. Abo-Shaeer, C. Raman, J. M. Vogels, and W. Ketterle, Observation of vortex lattices in Bose-Einstein condensates, Science, 292 (2001), pp. 476-479.

[2] S. K. Adhikari, Numerical study of the coupled time-dependent Gross-Pitaevskii equation: Application to Bose-Einstein condensation, Phy. Rev. E, 63 (2001), article 056704.

[3] G. Akrivis, Finite difference discretization of the cubic Schrödinger equation, IMA J. Numer. Anal., 13 (1993), pp. 115-124. MR1199033 (94d:65046)

[4] G. Akrivis, V. Dougalis and O. Karakashian, On fully discrete Galerkin methods of second-order temporal accuracy for the nonlinear Schrödinger equation, Numer. Math., 59 (1991), pp. 31-53. MR1103752 (92a:65256)

[5] M. H. Anderson, J. R. Ensher, M. R. Matthewa, C. E. Wieman and E. A. Cornell, Observation of Bose-Einstein condensation in a dilute atomic vapor, Science, 269 (1995), pp. 198-201.

[6] W. Bao, Q. Du and Y. Zhang, Dynamics of rotating Bose-Einstein condensates and its efficient and accurate numerical computation, SIAM J. Appl. Math., 66 (2006), pp. 758-786. MR2216159 (2006k:35267)

[7] W. Bao, D. Jaksch and P. A. Markowich, Numerical solution of the Gross-Pitaevskii equation for Bose-Einstein condensation, J. Comput. Phys., 187 (2003), pp. 318-342. MR.1977789 (2004g:82065)

[8] W. Bao, S. Jin and P. A. Markowich, On time-splitting spectral approximation for the Schrödinger equation in the semiclassical regime, J. Comput. Phys., 175 (2002), pp. 487-524. MR.1880116

[9] W. Bao, H. Li and J. Shen, A generalized-Laguerre-Fourier-Hermite pseudospectral method for computing the dynamics of rotating Bose-Einstein condensates, SIAM J. Sci. Comput., 31 (2009), pp. 3685-3711. MR2556558 (2010j:65193)

[10] W. Bao and J. Shen, A Fourth-order time-splitting Laguerre-Hermite pseudo-spectral method for Bose-Einstein condensates, SIAM J. Sci. Comput., 26 (2005), pp. 2010-2028. MR2196586 (2006i:65177)

[11] W. Bao and H. Wang, An efficient and spectrally accurate numerical method for computing dynamics of rotating Bose-Einstein condensates, J. Comput. Phys., 217 (2006), pp. 612-626. MR2260616 (2007k:82132)

[12] W. Bao, H. Wang and P. A. Markowich, Ground, symmetric and central vortex states in rotating Bose-Einstein condensates, Commun. Math. Sci., 3 (2005), pp. 57-88. MR:2132826 (2006b:82013)

[13] C. Besse, B. Bidégaray and S. Descombes, Order estimates in time of splitting methods for the nonlinear Schrödinger equation, SIAM J. Numer. Anal., 40 (2002), pp. 26-40. MR 1921908 (2003k:65099)

[14] B. M. Caradoc-Davis, R. J. Ballagh and K. Burnett, Coherent dynamics of vortex formation in trapped Bose-Einstein condensates, Phys. Rev. Lett., 83 (1999), pp. 895-898.

[15] T. Cazenave, Semilinear Schrödinger equations, (Courant Lecture Notes in Mathematics vol. 10), New York University, Courant Institute of Mathematical Sciences, AMS, 2003. 
[16] Q. Chang, B. Guo and H. Jiang, Finite difference method for generalized Zakharov equations, Math. Comp., 64 (1995), pp. 537-553. MR.1284664 (95f:65163)

[17] Q. Chang, E. Jia and W. Sun, Difference schemes for solving the generalized nonlinear Schrödinger equation, J. Comput. Phys., 148 (1999), pp. 397-415. MR1669707 (99i:65086)

[18] K. B. Davis, M. O. Mewes, M. R. Andrews, N. J. van Druten, D. S. Durfee, D. M. Kurn and W. Ketterle, Bose-Einstein condensation in a gas of sodium atoms, Phys. Rev. Lett., 75 (1995), pp. 3969-3973.

[19] A. Debussche and E. Faou, Modified energy for split-step methods applied to the linear Schrödinger equations, SIAM J. Numer. Anal., 47 (2009), pp. 3705-3719. MR2576517 (2010m:65169)

[20] C. M. Dion and E. Cances, Spectral method for the time-dependent Gross-Pitaevskii equation with a harmonic trap, Phys. Rev. E, 67 (2003), article 046706.

[21] R. T. Glassey, Convergence of an energy-preserving scheme for the Zakharov equations in one space dimension, Math. Comp., 58 (1992), pp. 83-102. MR1106968 (92e:65123)

[22] B. Y. Guo, The convergence of numerical method of nonlinear Schrödinger equation, J. Comput. Math., 4 (1986), 121-130. MR854389 (89a:65136)

[23] C. Hao, L. Hsiao and H. Li, Global well posedness for the Gross-Pitaevskii equation with an angular momentum rotational term in three dimensions, J. Math. Phys., 48 (2007), article 102105. MR2362770 (2008m:35336)

[24] C. Hao, L. Hsiao and H. Li, Global well posedness for the Gross-Pitaevskii equation with an angular momentum rotational term, Math. Meth. Appl. Sci., 31 (2008), pp. 655-664. MR2400070 (2009f:35317)

[25] R. H. Hardin and F. D. Tappert, Applications of the split-step Fourier method to the numerical solution of nonlinear and variable coefficient wave equations, SIAM Rev. Chronicle, 15 (1973), pp. 423.

[26] O. Karakashian, G. Akrivis and V. Dougalis, On optimal order error estimates for the nonlinear Schrödinger equation, SIAM J. Numer. Anal., 30 (1993), pp. 377-400. MR.1211396 (94c:65119)

[27] R. Landes, On Galerkin's method in the existence theory of quasilinear elliptic equations, J. Funct. Anal., 39 (1980), pp. 123-148. MR597807 (83j:35064)

[28] M. Lees, Approximate solution of parabolic equations, J. Soc. Indust. Appl. Math., 7 (1959), pp. 167-183. MR0110212 (22:1092)

[29] E. H. Lieb and R. Seiringer, Derivation of the Gross-Pitaevskii equation for rotating Bose gases, Commun. Math. Phys., 264 (2006), pp. 505-537. MR2215615(2007h:82008)

[30] C. Lubich, On splitting methods for Schrödinger-Poisson and cubic nonlinear Schrödinger equations, Math. Comp., 77 (2008), pp. 2141-2153. MR2429878(2009d:65114)

[31] P. A. Markowich, P. Pietra and C. Pohl, Numerical approximation of quadratic observables of Schrödinger-type equations in the semi-classical limit, Numer. Math., 81 (1999), pp. 595-630. MR 1675220 (2000a:81038)

[32] M. R. Matthews, B. P. Anderson, P. C. Haljan, D. S. Hall, C. E. Wiemann and E. A. Cornell, Vortices in a Bose-Einstein condensate, Phys. Rev. Lett., 83 (1999), pp. 2498-2501.

[33] C. Neuhauser and M. Thalhammer, On the convergence of splitting methods for linear evolutionary Schrödinger equations involving an unbounded potential, BIT, 49 (2009), pp. 199-215. MR2486135(2009m:65163)

[34] K. Ohannes and M. Charalambos, A space-time finite element method for the nonlinear Schrödinger equation: the continuous Galerkin method, SIAM J. Numer. Anal., 36 (1999), pp. 1779-1807. MR.1712169 (2000h:65139)

[35] L. P. Pitaevskii and S. Stringari, Bose-Einstein Condensation, Clarendon Press, Oxford, 2003. MR2012737 (2004i:82038)

[36] M. P. Robinson, G. Fairweather and B. M. Herbst, On the numerical solution of the cubic Schrödinger equation in one space variable, J. Comput. Phys., 104 (1993), pp. 277-284. MR.1198234

[37] T. R. Taha and M. J. Ablowitz, Analytical and numerical aspects of certain nonlinear evolution equations, II. Numerical, nonlinear Schrödinger equation, J. Comput. Phys., 55 (1984), pp. 203-230. MR762363(86e:65128b) 
[38] M. Thalhammer, High-order exponential operator splitting methods for time-dependent Schrödinger equations, SIAM J. Numer. Anal., 46 (2008), pp. 2022-2038. MR 2399406 (2009b:65163)

[39] M. Thalhammer, M. Caliari and C. Neuhauser, High-order time-splitting Hermite and Fourier spectral methods, J. Comput. Phys., 228 (2009), pp. 822-832. MR 2477790 (2010e:65179)

[40] V. Thomée, Galerkin finite element methods for parabolic problems, Springer, 1997. MR 1479170 (98m:65007)

[41] Y. L. Zhou, Implicit difference scheme for the generalized non-linear Schrödinger system, J. Comput. Math., 1 (1983), 116-129.

Department of Mathematics and Center for Computational Science and Engineering, National University of Singapore, Singapore 119076

E-mail address: bao@math.nus.edu.sg

$U R L:$ http://www.math.nus.edu.sg/ bao/

Department of Mathematics, National University of Singapore, Singapore 119076

E-mail address: caiyongyong@nus.edu.sg 